\title{
Influenza-mediated reduction of lung epithelial ion channel activity leads to dysregulated pulmonary fluid homeostasis
}

\author{
Jeffrey D. Brand, ${ }^{1}$ Ahmed Lazrak, ${ }^{1}$ John E. Trombley, ${ }^{1}$ Ren-Jay Shei, ${ }^{2,3}$ A. Timothy Adewale, ${ }^{3}$ \\ Jennifer L. Tipper, ${ }^{1}$ Zhihong Yu, ${ }^{1}$ Amit R. Ashtekar, ${ }^{1}$ Steven M. Rowe, ${ }^{2,3}$ Sadis Matalon, ${ }^{1}$ \\ and Kevin S. Harrod ${ }^{1}$ \\ 'Department of Anesthesiology and Perioperative Medicine, Division of Molecular and Translational Biomedicine, \\ ${ }^{2}$ Department of Medicine, Division of Pulmonary, Allergy, and Critical Care, and ${ }^{3}$ Gregory Fleming James Cystic Fibrosis \\ Research Center, School of Medicine, University of Alabama at Birmingham, Birmingham, Alabama, USA.
}

\begin{abstract}
Severe influenza (IAV) infection can develop into bronchopneumonia and edema, leading to acquired respiratory distress syndrome (ARDS) and pathophysiology. Underlying causes for pulmonary edema and aberrant fluid regulation largely remain unknown, particularly regarding the role of viral-mediated mechanisms. Herein, we show that distinct IAV strains reduced the functions of the epithelial sodium channel $(\mathrm{ENaC})$ and the cystic fibrosis transmembrane regulator (CFTR) in murine respiratory and alveolar epithelia in vivo, as assessed by measurements of nasal potential differences and single-cell electrophysiology. Reduced ion channel activity was distinctly limited to virally infected cells in vivo and not bystander uninfected lung epithelium. Multiple lines of evidence indicated ENaC and CFTR dysfunction during the acute infection period; however, only CFTR dysfunction persisted beyond the infection period. ENaC, CFTR, and Na,K-ATPase activities and protein levels were also reduced in virally infected human airway epithelial cells. Reduced $\mathrm{ENaC}$ and CFTR led to changes in airway surface liquid morphology of human tracheobronchial cultures and airways of IAV-infected mice. Pharmacologic correction of CFTR function ameliorated IAV-induced physiologic changes. These changes are consistent with mucous stasis and pulmonary edema; furthermore, they indicate that repurposing therapeutic interventions correcting CFTR dysfunction may be efficacious for treatment of IAV lung pathophysiology.
\end{abstract}

Authorship note: JDB and AL contributed equally as co-first author. SM and KSH contributed equally as co-senior author.

Conflict of interest: SM reports income from the American Physiological Society for his role as editor in chief of Physiological Reviews.

License: Copyright 2018, American Society for Clinical Investigation.

Submitted: July 13, 2018 Accepted: September 6, 2018 Published: October 18, 2018

\section{Reference information:} JCI Insight. 2018;3(20):e123467. https://doi.org/10.1172/jci. insight.123467.

\section{Introduction}

Acquired respiratory distress syndrome (ARDS) secondary to lower respiratory tract infections, particularly to viruses, remains a serious clinical outcome following infection. Upward of $10 \%$ of admissions to intensive care settings during the typical influenza (IAV) season are for pneumonia or ARDS requiring respiratory support (1-3). Viral-mediated pneumonia is recognized across most common respiratory viral infections, and with the lack of broad-spectrum antiviral therapies, treatment is largely supportive and nonspecific. Targeted therapies addressing pulmonary edema and poor ventilatory function during infection are not available and are hampered by a poor understanding of the underlying molecular events that confer epithelial fluid regulation. A clearer understanding of the underlying pathobiology of fluid barrier function of the respiratory epithelium is urgently needed.

Proper lung function requires a hydrated airway epithelium to facilitate mucociliary clearance and conductance of air, while the alveolar epithelium must be essentially devoid of water for oxygen to diffuse efficiently for gas exchange. Pulmonary fluid regulation is mediated by a complex interplay of epithelial tight junctions and secretion and reabsorption of transcellular ions, such as $\mathrm{Na}^{+}, \mathrm{Cl}^{-}, \mathrm{HCO}_{3}^{-}$, and $\mathrm{K}^{+}$, to which water travels passively along osmotic gradients (4-6). These ions move passively through channels and transporters, down a favorable electrochemical gradient established by the energy-consuming, ouabain-sensitive, Na,K-ATPase located in the basolateral surface (5). Loss of barrier properties and/or dysregulation of $\mathrm{Na}^{+}, \mathrm{Cl}^{-}, \mathrm{K}^{+}$gradients across the epithelial mucosa can independently reduce the airway 
and alveolar fluid along the apical epithelial surfaces $(7,8)$. A number of notable diseases of ion channel function, such as cystic fibrosis (CF), lead to dysregulated fluid levels in various lung compartments, and these diseases are often associated with pulmonary infection (9-11).

Upon infection of the host lung epithelium, the IAV virus can mediate a number of aberrant host cell responses, some on the part of the virus to manipulate the host cellular machinery to facilitate viral replication, release, and transmission and other responses invoked by the host for cellular host defense and protection of neighboring, uninfected epithelium. The effect of the viral-mediated and host-mediated events on the rheologic physiologic function of the lung epithelium remain largely unexplored. Herein, we show through what we believe to be the novel application of electrophysiological and imaging techniques that IAV regulation of ion channel dysfunction indeed can be found in primary human and mouse airway epithelia. Furthermore, the regulation of epithelial sodium channel $(\mathrm{ENaC})$ and cystic fibrosis transmembrane regulator (CFTR) can be observed only in IAV-infected cells and for an extended period beyond the period of active viral replication. Using what we believe to be novel optical imaging techniques, changes in the airway surface fluid (ASL) and airway mucus responses, both airway responses that are mediated by reduced ion channel function, were concordantly altered in the airway pathophysiology to IAV infection in vivo. Collectively, our findings provide the first evidence to our knowledge in intact airway epithelium and in vivo that IAV-mediated regulation of ion channel function contributes to the pathogenesis of IAV infection.

\section{Results}

Ion channel function after IAV in vivo. Using an intact in vivo murine model of IAV infection, we measured $\mathrm{ENaC}$ and CFTR activities by performing measurements of nasal potential differences (NPD) in the presence of $\mathrm{ENaC}$ and CFTR inhibitors at various time points following infection. NPD measurements provide insight into specific ion channel activity in the upper respiratory tract and may be a surrogate for technically difficult measurements within the lower respiratory tract (12). Continuous measurement tracings of electrical potentials prior to and following application of specific inhibitors of ENaC and CFTR across upper respiratory tract epithelium at representative time points following IAV infection are shown in Figure 1A. The amiloride-inhibited portion of NPD, a measure of $\mathrm{Na}^{+}$transport across ENaC, was significantly decreased at days 5 and 10 after infection (p.i.) (Figure 1B) but returned to and exceeded control levels by day 15 p.i. The GlyH-101 inhibitable portion of NPD, a measure of CFTR activity, was also significantly decreased by days 5,10 , and 15 p.i.; however, in contrast to ENaC, the CFTR portion of NPD remained decreased at 15 days p.i. (Figure 1C). Taken altogether, these data demonstrate that in vivo IAV infection reduces $\mathrm{Na}^{+}$absorption (through $\mathrm{ENaC}$ ) and $\mathrm{Cl}^{-} / \mathrm{HCO}_{3}{ }^{-}$secretion (through CFTR) across mouse nasal epithelium transiently and consistently. This phenotype resembles that seen in patients with $\mathrm{CF}$ and COPD (13), which are known to be associated with altered airway rheology and mucociliary defects $(14,15)$.

Ion channel function dysfunction occurs only in cells with active infection. To provide further evidence of ion channel dysfunction in vivo after IAV infection and establish dependence on viral infection per se rather than paracrine factors or secondary events, C57BL/6 mice were infected intranasally with a nonlethal dose of GFP-tagged IAV A/Puerto Rico/8/1934 ${ }^{\triangle \mathrm{GFP}}\left(\mathrm{PR} / 8^{\triangle \mathrm{GFP}}\right)$. ENaC activity was measured in alveolar type II (AT2) cells by the cell-attached patch-clamp technique in lung tissue slices at selected time points following IAV infection, as described previously $(16,17)$. GFP fluorescence allowed the localization of infected cells, and ENaC activity was recorded from both infected (Figure 2, A and B) and noninfected cells from the same preparations. Recordings from unstained AT2 cells shows the baseline $\mathrm{ENaC}$ activity; channels with conductances of 4 and $18 \mathrm{pS}$ each were seen with open probabilities of 0.3 and 0.22 , respectively (Figure 2, C and D), similar to what we have reported previously $(14,15)$. When $\mathrm{ENaC}$ activity was recorded by patch clamp from $\mathrm{GFP}^{+}$cells, a marked reduction in the activity of both channels was observed (Figure 2, E and F). Patch opening probabilities of the 4 and $18 \mathrm{pS}$ channels, as assessed across the time course of IAV infection, are shown for GFP ${ }^{-}$and $\mathrm{GFP}^{+}$AT2 cells (Figure 3). In $\mathrm{GFP}^{+} \mathrm{AT} 2$ cells, the open probabilities of both the 4 and $18 \mathrm{pS}$ channels were significantly reduced starting at 2 days p.i.; they remained decreased, while returning slightly at day 15 , when virus was not detectable within lung tissue (Figure 3, A and B). In contrast, recordings from $\mathrm{GFP}^{-}$cells in the same lung slices showed normal values (Figure 3, C and D) through the infection period. These data suggest that IAV infection is necessary to decrease $\mathrm{ENaC}$ channel activity, with paracrine factors playing a lesser role or no role (18-20). We stress that ENaC activities in cell-attached patches are independent of $\mathrm{Na}, \mathrm{K}$-ATPase activity and thus demonstrate that IAV alters ENaC activity in vivo. 
A

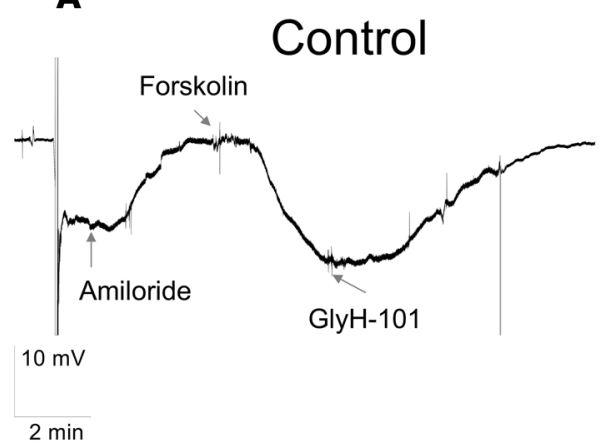

5 Day Infection

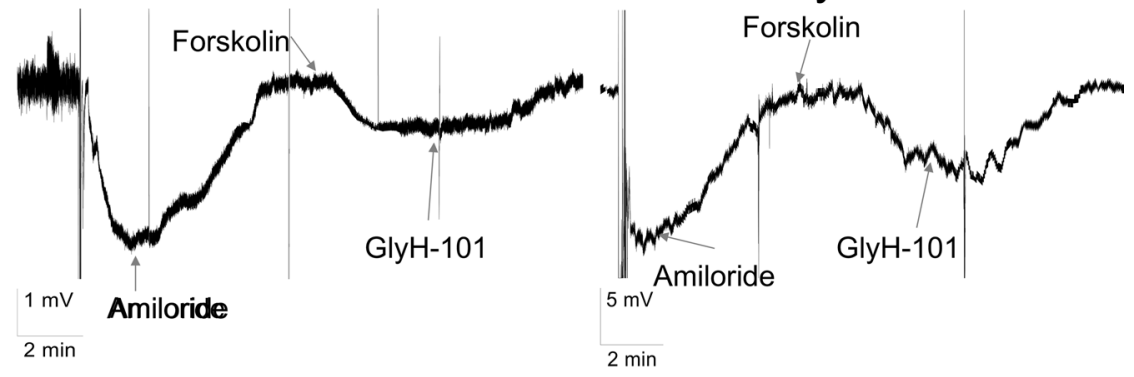

B

\section{Sodium Dependent Amiloride inhibited NPD}

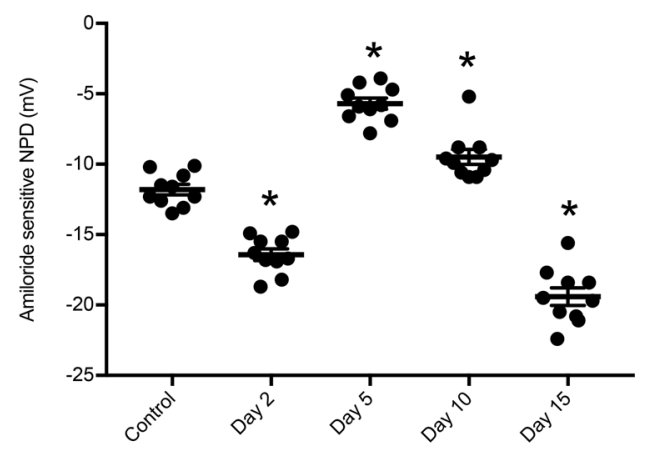

C Chloride Dependent GlyH-101 inhibited NPD

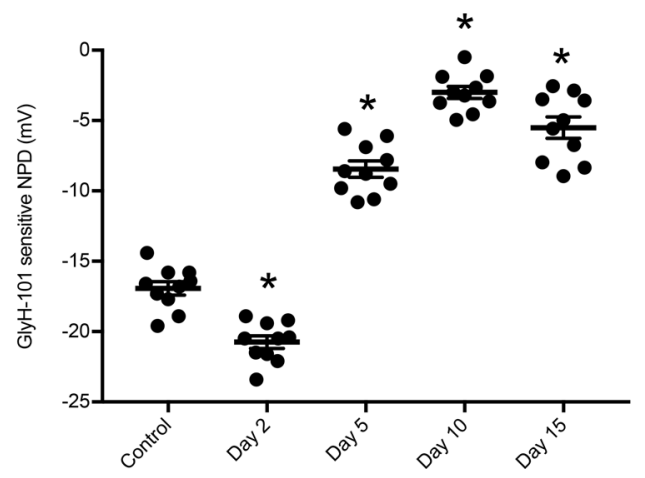

Figure 1. IAV infection reduces ENaC and CFTR function in vivo. Mice were infected with 4,000 PFU of PR8 ${ }^{\triangle C F P}$. NPD of control and infected mice were measured as described in Methods. (A) Recording traces depicting NPD in noninfected control mice, immediately after the probe was inserted in one the mouse nares; a negative voltage deflection was recorded and is indicative of the active $\mathrm{Na}^{+}$ion absorption. Addition of $200 \mu \mathrm{M}$ amiloride in the perfusate inhibited the voltage difference, indicating the role of ENaC in maintaining the initially recorded NPD. Subsequent perfusion with 10-20 $\mu \mathrm{M}$ forskolin activated a second potential difference due to the movement of $\mathrm{Cl}^{-}$and $\mathrm{HCO}_{3}^{-}$, which was inhibited by $50 \mu \mathrm{M}$ GlyH-101, showing the role of CFTR in this process. Representative NPD recordings from uninfected (Control) mice, 5 and 15 days p.i. are shown, with the electrical potential scale noted for each time point (bottom left). (B) ENaC activity, as measured by NPD across time course of IAV. Scatter plots with NPD value \pm SEM at 2, 5, 10, and 15 days p.i. (C) Scatter plots of GlyH-101-sensitive (CFTR) NPD at days 2, 5, 10, and 15 p.i. Each data point corresponds to a single mouse, $N=9-13$ per group; mean \pm SEM. Significance was determined by 1-way ANOVA and post hoc Tukey test for multiple comparisons. ${ }^{*} P<0.0001$ compared with noninfected controls.

CFTR channel activity is integral in the pathogenesis of airway diseases, such as CF and chronic bronchitis. CFTR channel activity in AT2 cells, even after forskolin stimulation, is difficult to detect most likely due to the small amount of activated protein (21). Therefore, single-cell patch-clamp experiments for CFTR activity were conducted on infected (fluorescent) or noninfected (nonfluorescent) normal human bronchial epithelial cells (NHBEs) grown on glass coverslips 48 hours following infection with PR/8 $8^{\Delta \mathrm{GFP}}$. As shown in Figure 4 , A and $\mathrm{B}, \mathrm{CFTR}$ function was readily measured by this technique with a single conductance of about $6 \mathrm{pS}$, similar to that reported for CFTR $(22,23)$. After IAV infection, single-channel activity was reduced considerably (Figure 4, C and D) without affecting the channel's conductance. Figure 4E shows open probabilities of CFTR in $\mathrm{GFP}^{-}$and $\mathrm{GFP}^{+}$NHBEs following IAV infection. Altogether, these data demonstrate that in vivo and in vitro IAV infection results in decreased of lung epithelial $\mathrm{ENaC}$ and CFTR function at the single-channel level and that the decrease in channel activities persists through the duration of active infection and beyond.

IAV infection decreases short-circuit current across human airway epithelial cells. Primary epithelial cultures of human or mouse tracheobronchial cells can develop many mucosal characteristics driven by the functional activity of ion channels in apical membranes. Previous reports have established IAV regulation of ENaC and CFTR in transformed cell lines $(24,25)$. Measurements of short-circuit current assess vectorial transport across epithelial cells, which depends on the coordinated action of Na,K-ATPase, basolateral channels, and transporters (such as $\mathrm{K}^{+}$channels and the $\mathrm{Na}-\mathrm{K}-\mathrm{Cl}$ cotransporter) as well as apical ENaC and CFTR. NHBEs were grown at an air-liquid interface, and once they formed confluent monolayers, they were infected with 
A

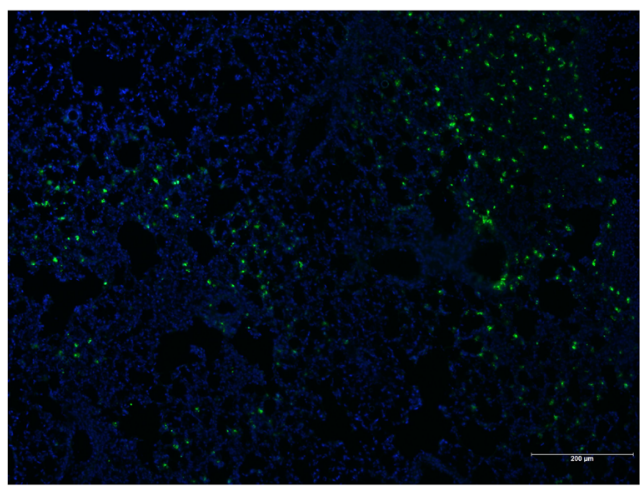

B

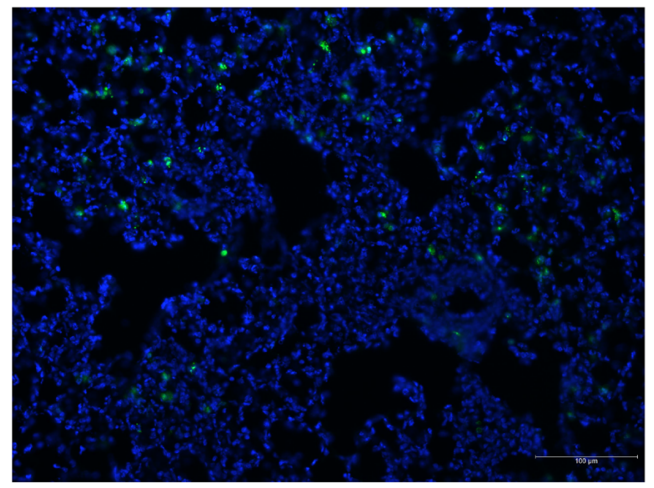

C

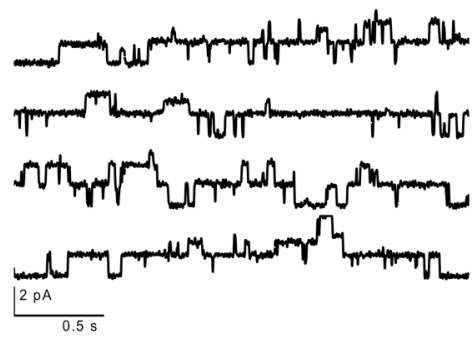

$\mathbf{E}$

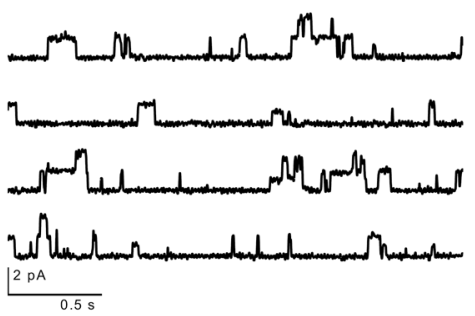

D

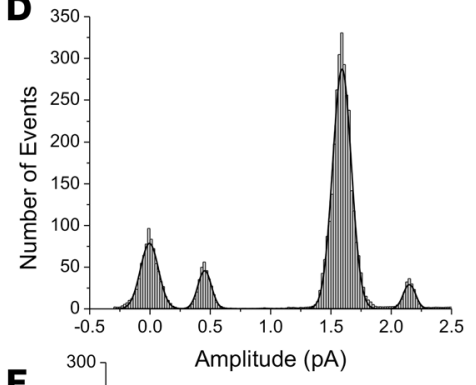

$\mathbf{F}$

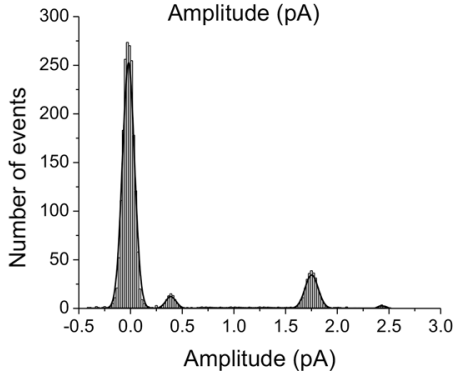

Figure 2. IAV reduces ENaC single-channel activity in infected but not in uninfected cells. Mice were infected with 4,000 PFU of PR8 ${ }^{\triangle C F P}$, lungs were harvested 5 days p.i., and fresh $250 \mu \mathrm{M}$ slices were prepared using a live tissue slicer from uninfected controls and infected animals as previously described (14). Slices were incubated in culture medium for 3-4 hours before ion channel activity was recorded from GFP+ and GFP- cells. Lung cryosections display widespread infection. GFP- cells exhibited single channels with conductance of $4 \mathrm{pS}$ and $18 \mathrm{pS}$. Recordings were obtained at $\mathrm{V}_{\text {patch }}+$ $100 \mathrm{mV}$ when $V_{m}$ was $0 \mathrm{mV}$ (slices incubated in $135 \mathrm{mM} \mathrm{KCl}$ Ringer). Original magnification, $\times 100$ (A) and $\times 200$ (B). (C) Tracings of ENaC open probability conductance in GFP- cells. Histogram shown in $\mathbf{D}$ shows number of events for the 4 Ps channel only. (E) A representative recording of channel activities recorded from GFP+-infected AT2 cells. (F) GFP+ AT2 cells exhibited substantial reduction of $4 \mathrm{pS}$ and $18 \mathrm{pS}$ activity, without affecting their unitary conductances. Please see Figure 3 for quantification of these findings.

2009 pandemic H1N1 IAV. Short-circuit currents and transepithelial resistances were measured in Ussing chambers across intact and permeabilized monolayers $24-72$ hours p.i., as described previously (26, 27). As shown in Figure 5A, amiloride-sensitive (ENaC) and forskolin-stimulated GlyH-101-inhibited (CFTR) currents were not altered at 24 hours p.i. However, at 48 and 72 hours p.i., ENaC and CFTR currents were reduced significantly $(P<0.0001)$ (Figure $5, \mathrm{~B}$ and $\mathrm{C})$. This effect was also seen when NHBEs were infected with the mouse-adapted PR/8 IAV virus (data not shown), indicating that reductions of ion channel activity are not limited to 2009 pandemic H1N1. These findings are consistent with our in vivo data and show that ion channel dysfunction in individual cells is sufficient to alter the epithelial barrier ion transport.

Transepithelial electrical resistance (TEER) of the cellular monolayers was also recorded. Neither 2009 pdm H1N1 or PR/8 IAV strains reduced monolayer resistance 24 hours p.i (Figure 6). However, both strains significantly decreased monolayer resistance at 48 hours p.i. Though these decreases were significant, they did not cause a breakdown of the cellular monolayers, indicating that most cells were still intact and functional at 48 hours p.i. and that TEER was sufficient to obtain experimental short-circuit measurements by electrophysiology approaches. These data indicate that IAV infection can reduce TEER due to IAV infection that is not a result of significant damage to the cell monolayer. 

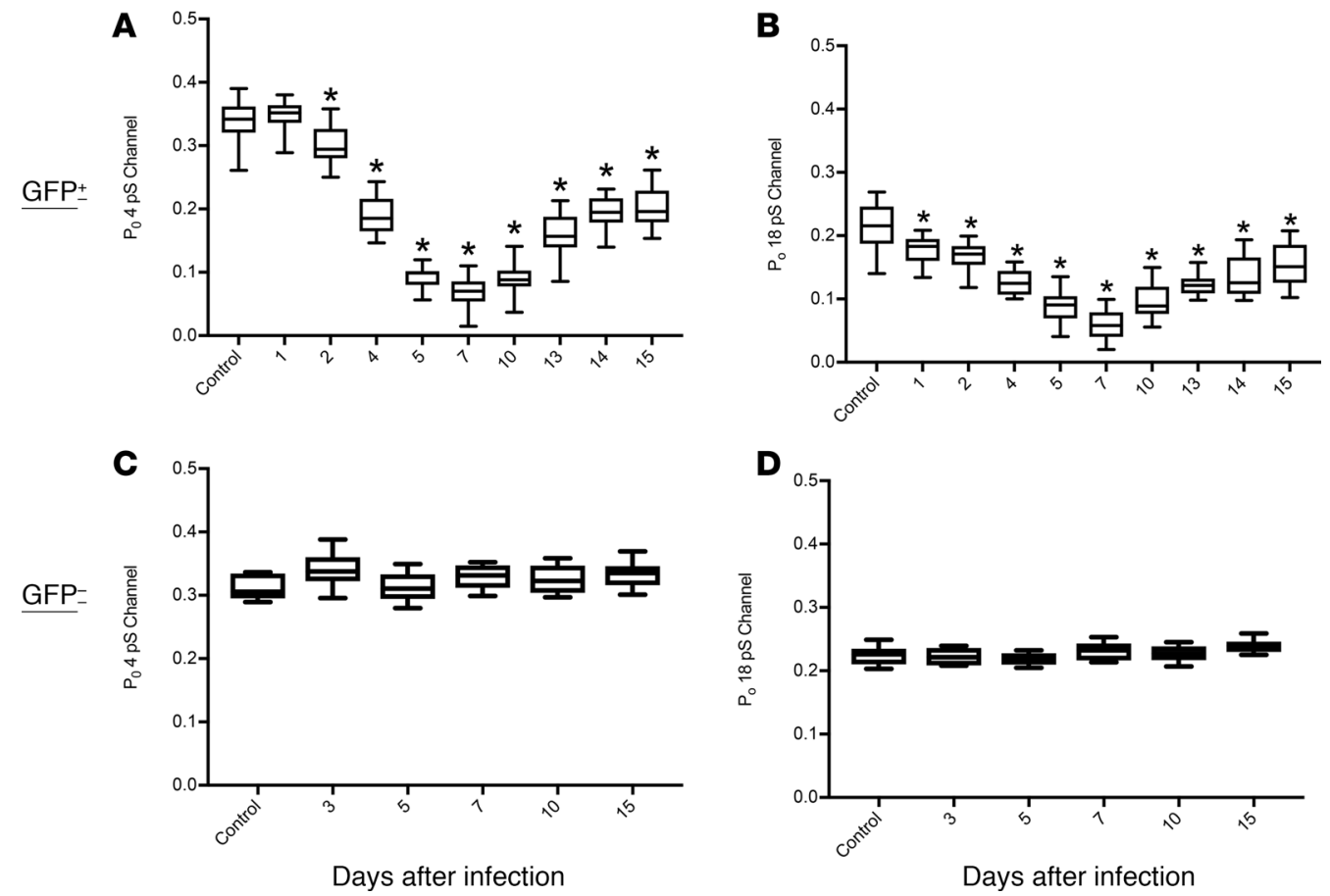

Figure 3. ENaC channel dysfunction during IAV infection. Mice were infected with $4,000 \mathrm{PFU}$ of PR8 $8^{\triangle C F P}$; lungs were harvested at days 1, 2, 4, 5, 7, 10, 13, 14, and 15 p.i.; and live slices were cut using a live tissue slicer. ENaC activities in infected AT2 cells (GFP) were recorded using cell-attached mode of the patch-clamp technique. (A and $\mathbf{B})$ Reduction of $\mathrm{ENaC}$ open probabilities for various times after infection. $N=5$ mice and $n=29$ cells per group. The maximum inhibition was seen at day 7, after which a slow recovery of both conductances was observed. (C and D) ENaC activity, measured as open probability $\left(P_{0}\right)$, was not affected in noninfected cells. Data are plotted as mean open probabilities, top and bottom quartiles \pm SEM. Data were analyzed by 1-way ANOVA and post hoc Bonferroni correction for multiple comparisons. ${ }^{*} P<0.0001$ for each time point compared with noninfected control values.

In addition to measurements of ENaC and CFTR activity, the maximum $\mathrm{Na} / \mathrm{K}-\mathrm{ATPase}$ activity was also determined by permeabilizing the apical membranes with amphotericin $\mathrm{B}$, as described previously $(26,28)$. Representative short-circuit measurements of $\mathrm{Na} / \mathrm{K}$ ATPase activity at multiplicities of infection (MOIs) of 1-3 are shown (Figure 7A). IAV infection significantly reduced maximum $\mathrm{Na}, \mathrm{K}$-ATPase activity, with the greatest activity observed at higher MOI. Short-circuit measurements of multiple NHBE monolayers are shown in Figure 7B, indicating the consistent findings of reduced $\mathrm{Na} / \mathrm{K}$ ATPase activity following IAV infection.

To determine whether decreased $\mathrm{ENaC}, \mathrm{CFTR}$, and $\mathrm{Na}, \mathrm{K}$-ATPase activities were due to reductions in protein levels, NHBEs infected with IAV and $\alpha-, \beta-$, and $\gamma$-ENaC; CFTR; and the $\alpha$ and $\beta 1$ subunit of the $\mathrm{Na} / \mathrm{K}$-ATPase cellular protein abundances were assessed. Apical proteins were biotinylated for determination of cell surface protein expression, as detailed in Methods, as only cell surface expression can facilitate channel activity. As shown in Figure 8A, decreased levels of $\alpha-, \beta-, \gamma$-ENaC were noted at 48 hours p.i. Importantly, the $\beta$-ENaC subunit level was nearly completely ablated by this treatment. Surface-localized (biotin-labeled) CFTR C-band expression was also dramatically reduced after IAV infection. In addition, the $\alpha$ subunit of the $\mathrm{Na} / \mathrm{K}$-ATPase was also reduced, whereas there was no difference in expression of the $\beta 1$ subunit. Densitometry of multiple independent samples exhibited markedly decreased subunit abundance after IAV (Figure 8B). These data indicate that IAV infection downregulates ENaC, CFTR, and $\mathrm{Na} / \mathrm{K}$-ATPase ion channel cell surface abundance but does so in a subunit-specific manner.

IAV infection alters airway surface liquid rheology. The observed decreases in ion channel function led us to investigate whether airway surface liquid homeostasis was altered after IAV infection. To determine this, NHBEs were infected with 2009 pdm H1N1 IAV, and the cell monolayers were then imaged by micro-optical coherence tomography $(\mu \mathrm{OCT})$ for ASL depth and ciliary beat frequency $(\mathrm{CBF})(29,30)$. As shown in Figure 9B, IAV infection significantly decreased ASL depth 48 and 72 hours p.i. The decrease in ASL depth was independent of MOI. CBF was significantly decreased (Figure 9B) concurrent with changes in ASL 
A

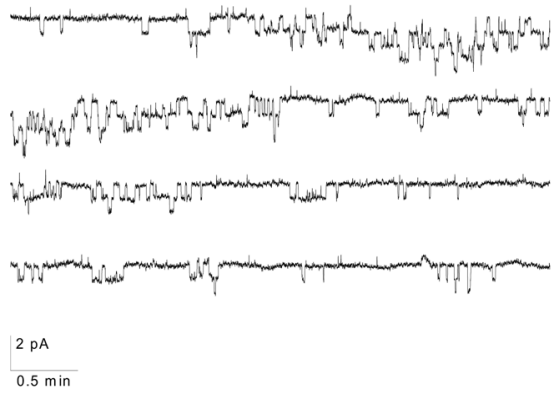

C

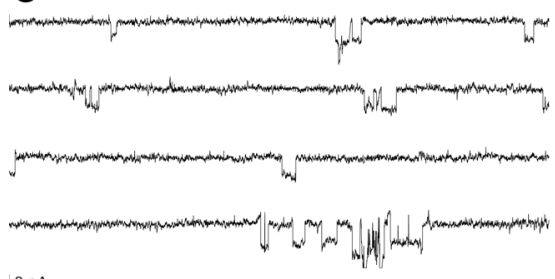

$2 \mathrm{pA}$

$0.5 \mathrm{~min}$

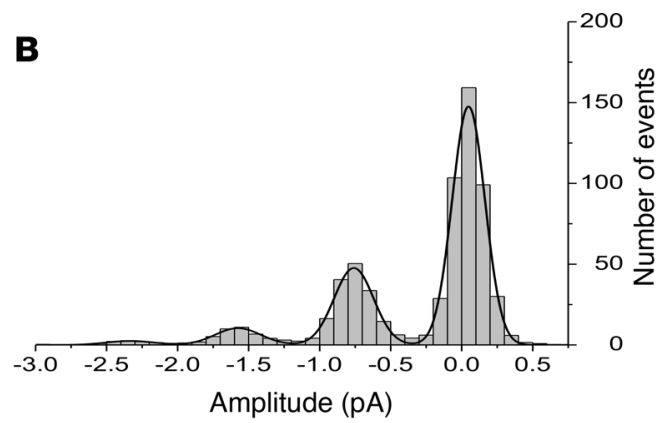

D

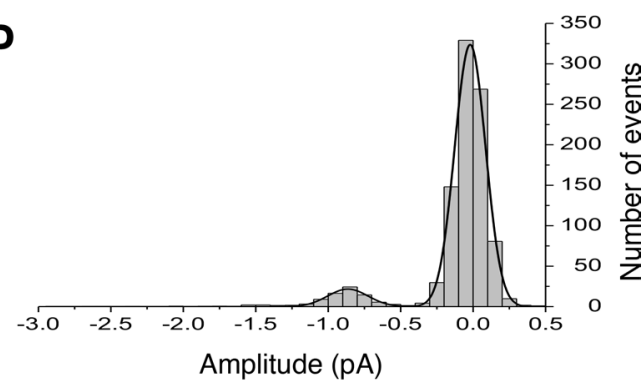

$\mathbf{E}$

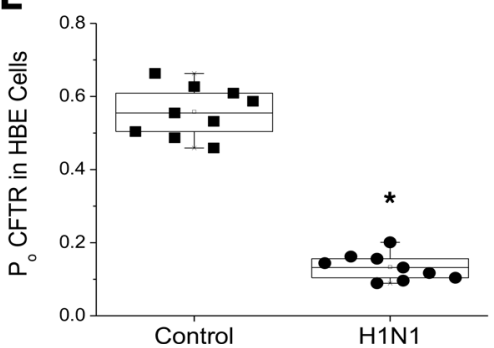

Figure 4. IAV reduces CFTR single-channel activity in infected but not uninfected NHBEs. NHBEs were grown on glass cover slips and infected with PR8 ${ }^{\triangle G F P}$ at a $\mathrm{MOI}$ of 6 . Infected (GFP+) and noninfected (GFP-) cells were patchclamped 48 hours p.i. in the cell-attached mode, and forskolin-treated single-channel activities (CFTR) were recorded. (A) Representative CFTR single-channel activity traces recorded from an uninfected NHBE. CFTR was activated with forskolin $(10 \mu \mathrm{M})$ and inhibited with PPQ-102 $(10 \mu \mathrm{M})$, a CFTR inhibitor. (B) Amplitude histogram showing the distribution of active channels after forskolin addition. Channel conductance recorded at $-100 \mathrm{mV}$ shows a peak at about 0.6 pA corresponding to a unitary conductance of $6 \mathrm{pS}$. (C and D) CFTR activity in NHBEs infected with PR8 ${ }^{\triangle \mathrm{ACF}}$. (C) Typical recording after addition of $10 \mu \mathrm{M}$ forskolin. (D) Forskolin activation of channel with $6 \mathrm{pS}$ conductance was reduced markedly in GFP+ cells. (E) CFTR activity, as open probability of the channels recorded from 9 uninfected and 9 infected cells. Data were analyzed by 1-way ANOVA followed by post hoc Tukey test for multiple comparisons. Data are plotted as mean values $(N=9)$, top and lower quartiles $\pm S D$. ${ }^{*} P<0.0001$.

depth as ASL depth can reduce $\mathrm{CBF}$ through changes in mechanical action of ciliary axonemes in a depleted surface fluid layer. The reduction in ASL again is significant, in that IAV-infected NHBEs can maintain an air-liquid barrier, indicating a viable airway epithelium. Representative topography images shown in Figure 9A indicate a depleted ASL layer, as noted by the reduced depth above the epithelial cell layer.

We next sought to determine whether the decrements of ion channel function noted also resulted in decreased ASL depth and CBF in vivo. Mice were infected with IAV, and $\mu \mathrm{OCT}$ imaging was performed on excised tracheas following IAV infection. A significant reduction in ASL depth was observed at day 4 p.i. (Figure 9D). CBF was also significantly reduced at 4 days p.i. (Figure 9D). Representative topography images of uninfected and IAV-infected tracheas show markedly depleted ASL following IAV infection (Figure 9C). These data demonstrate that in vivo IAV infection reduces the ASL depth in the airways and concomitantly reduces CBF, consistent with reduced CFTR function. These findings are strikingly similar to the airway pathophysiology observed in chronic airways diseases in which CFTR dysfunction is central to the molecular pathogenesis, such as disease in $C F(14,30,31)$ or acquired CFTR dysfunction in COPD $(15,32)$. 
A

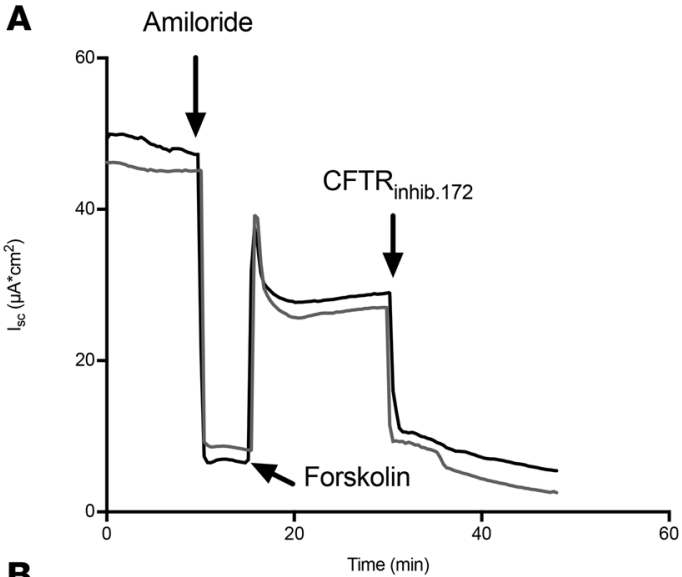

B
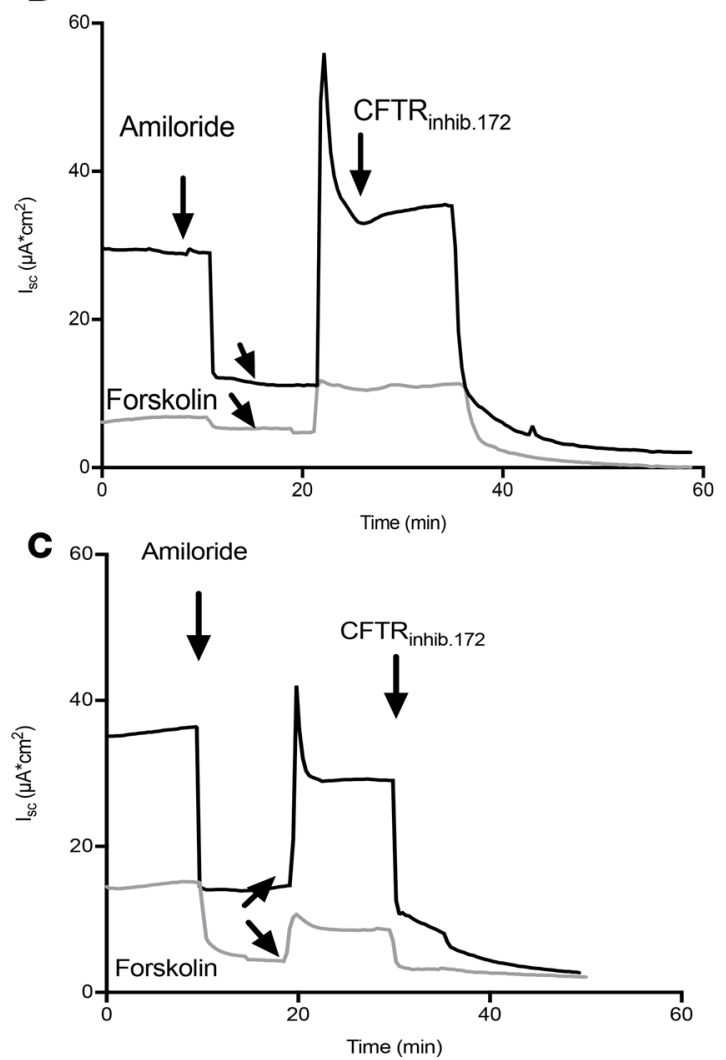
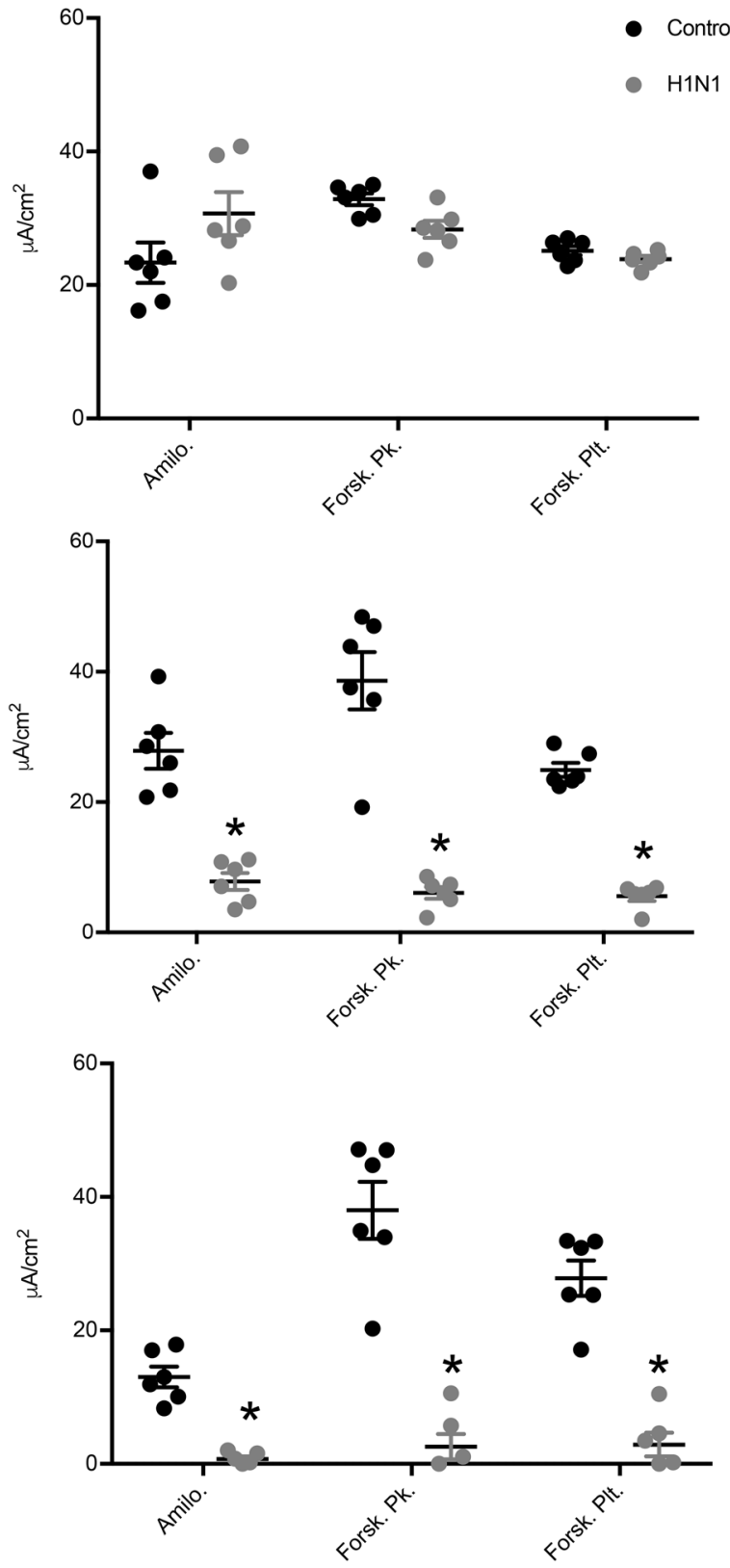

Figure 5. IAV reduces ENaC and CFTR short-circuit currents across intact normal human bronchial epithelial cell monolayers. NHBEs were grown at an air-liquid interface and infected with A/California/07/2009 H1N1 IAV at a MOI of 3 for (A) 24, (B) 48, and (C) 72 hours, at which point they were mounted in Ussing chambers for the measurement of short-circuit currents $\left(\mathrm{I}_{\mathrm{sc}}\right)$ and transepithelial resistance. Amiloride (ENaC) and forskolin-sensitive currents inhibited by the specific CFTR inhibitor CFTR $_{\text {inh172 }}$ were significantly reduced by IAV infection. Amilo., amiloride trough; Forsk Pk., forskolin peak; Forsk. Plt., forskolin plateau. Characteristic records $(\mathbf{A}-\mathbf{C})$ and scatter plots $(n=6$ filters per groups; mean \pm 1 SEM) (D-F). Data were analyzed by 1 -way ANOVA and post hoc Tukey test for multiple comparisons. ${ }^{*} P<0.0001$ compared with time-matched noninfected controls.

ASL homeostasis is restored by CFTR correction. The findings presented thus far indicate that IAV leads to physiologic changes consistent ASL depletion. The magnitude, duration, and loss of $\mathrm{Cl}^{-}$transport would be consistent with reduced ASL reduction, similar to that found experimentally with CF. Recently, a number of pharmacologic interventions targeting CFTR transit and correction of the CFTR $\mathrm{Cl}^{-}$ channel have been approved by the FDA (33). These drugs are also known to restore ASL homeostasis and restore partially physiologic function to $C F$ patients $(14,30)$. We conducted $\mu \mathrm{OCT}$ imaging of primary human airway epithelial cultures infected with IAV and treated with a compound targeting CFTR trafficking to the plasma membrane, specifically VX-809 (lumacaftor, Vertex Pharmaceuticals) (Figure 10A). Lumacaftor is a corrector of the CFTR protein, which improves CFTR processing and transport 
A

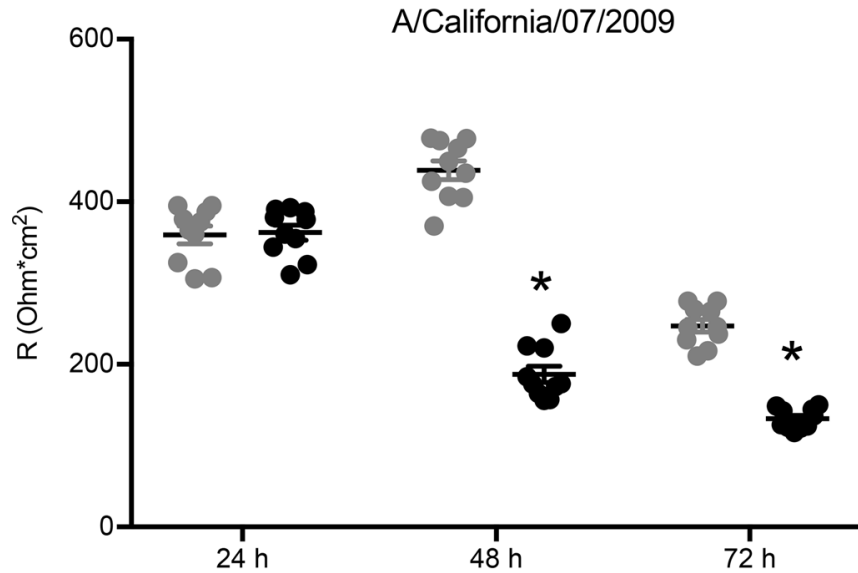

B

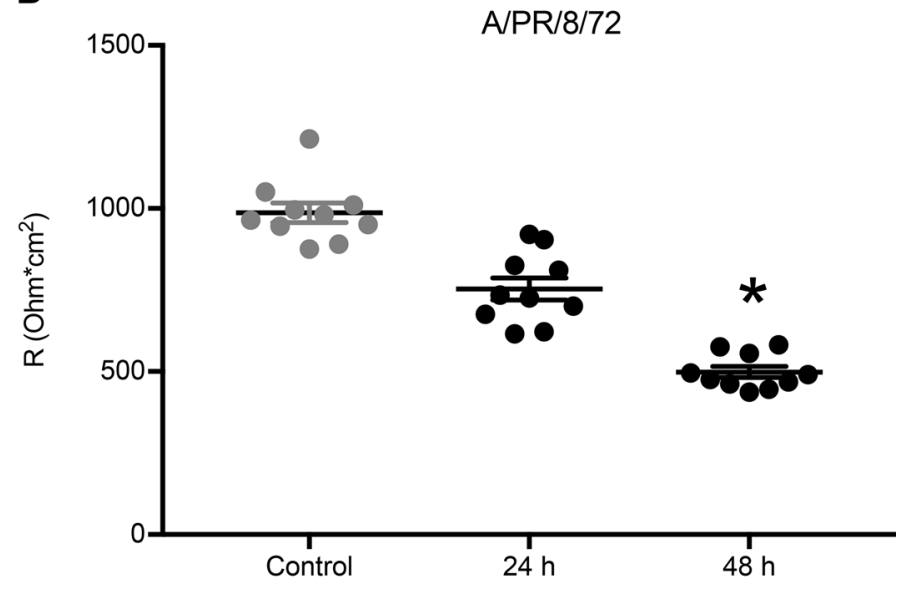

Figure 6. Transepithelial resistance is similarly decreased by two different strains of IAV. NHBEs were grown at an air-liquid interface and infected with (A) A/California/07/2009 or (B) A/PR/8/72 H1N1 IAV at a MOI of 3, and total transepithelial resistance was measured in an Ussing chamber system. Both IAV strains significantly reduced transepithelial resistance 48 hours p.i. Scatter plots, $n=10$, with mean \pm SEM. Data were analyzed by 1 -way ANOVA and post hoc Tukey test for multiple comparisons. ${ }^{*} P<0.0001$ compared with noninfected controls.

to the cell surface (34). As with previous experiments, ASL depth in NHBEs was typically observed at a depth of $10 \mu \mathrm{m}$, and IAV infection significantly reduced ASL depth to approximately $3 \mu \mathrm{m}$ (Figure 10B). Lumacaftor administration 1 hour following IAV infection was able to restore ASL depth to greater than $7 \mu \mathrm{m}$ following IAV. These findings support the role of diminished CFTR expression and function in IAV-mediated ASL loss and suggest that targeting CFTR correction may be a useful strategy in ameliorating IAV pathogenesis.

\section{Discussion}

The pathophysiology of IAV infection remains unresolved, particularly with regards to the underlying causes of lung disease and clinical presentation. The mucosal barrier of the conducting airways and integrity of the alveolar blood-gas exchange barrier are central to the sequelae of severe IAV infections, and investigations into the molecular mechanisms are urgently needed. Maintenance of the pulmonary mucosal barrier and gas exchange unit are critically dependent on intricate regulation of $\mathrm{Na}^{+}$absorption and $\mathrm{Cl}^{-}$and $\mathrm{HCO}_{3}^{-}$secretion via the ENaC and CFTR channels, respectively (reviewed in ref. 13). Previous studies have identified $\mathrm{ENaC}$ and CFTR channel dysfunction in response to IAV in various in vitro cell culture systems $(24,25,35)$. Herein, we have investigated ion channel dysfunction in human- and mouse-polarized, intact lung epithelium in a system that allows us to determine the effect of ion channel dysfunction on epithelial lining pathophysiology. Our findings show unequivocally the role of active IAV infection in reducing $\mathrm{ENaC}$ and CFTR function in polarized epithelium of both human and mouse experimental systems. Furthermore, using cutting edge imaging techniques, we show that ion channel dysfunction leads to airway pathophysiology that can be corrected by restoration of CFTR function. Finally, in vivo single-cell analysis of ion channel function indicates that ENaC and CFTR dysfunction is limited to only those cells infected with IAV.

NPD measurements are used clinically to determine ion channel function in individuals with suspected channelopathies and are an important clinical assessment tool of CFTR function in CF (36). Here, we have adapted this technology to investigate ENaC and CFTR dysfunction in murine IAV infection in vivo. Longitudinal studies across the course of infection indicated that $\mathrm{ENaC}$ activity is diminished at the peak of viral infection (typically days 5-7 p.i.) but returns to normal levels at the time of resolution of IAV disease. Our study supports the findings of Chen et al. (35) and Wolk et al. (37), who noted that reduced lung fluid clearance after IAV infection resulted from decreases in ENaC activity mediated by IAV. CFTR is decreased to an even greater magnitude at the peak of viral infection; however, CFTR remains markedly decreased up to 15 days p.i. Downregulation of CFTR was also noted by Chan et al. (38) after IAV infection, with both $\mathrm{H} 1 \mathrm{~N} 1$ and $\mathrm{H} 5 \mathrm{~N} 1$ strains, in agreement with our findings using distinct IAV strains, while Wolk et al. (37) noted a rise in CFTR activity after IAV infection via alveolar fluid clearance measurements. 

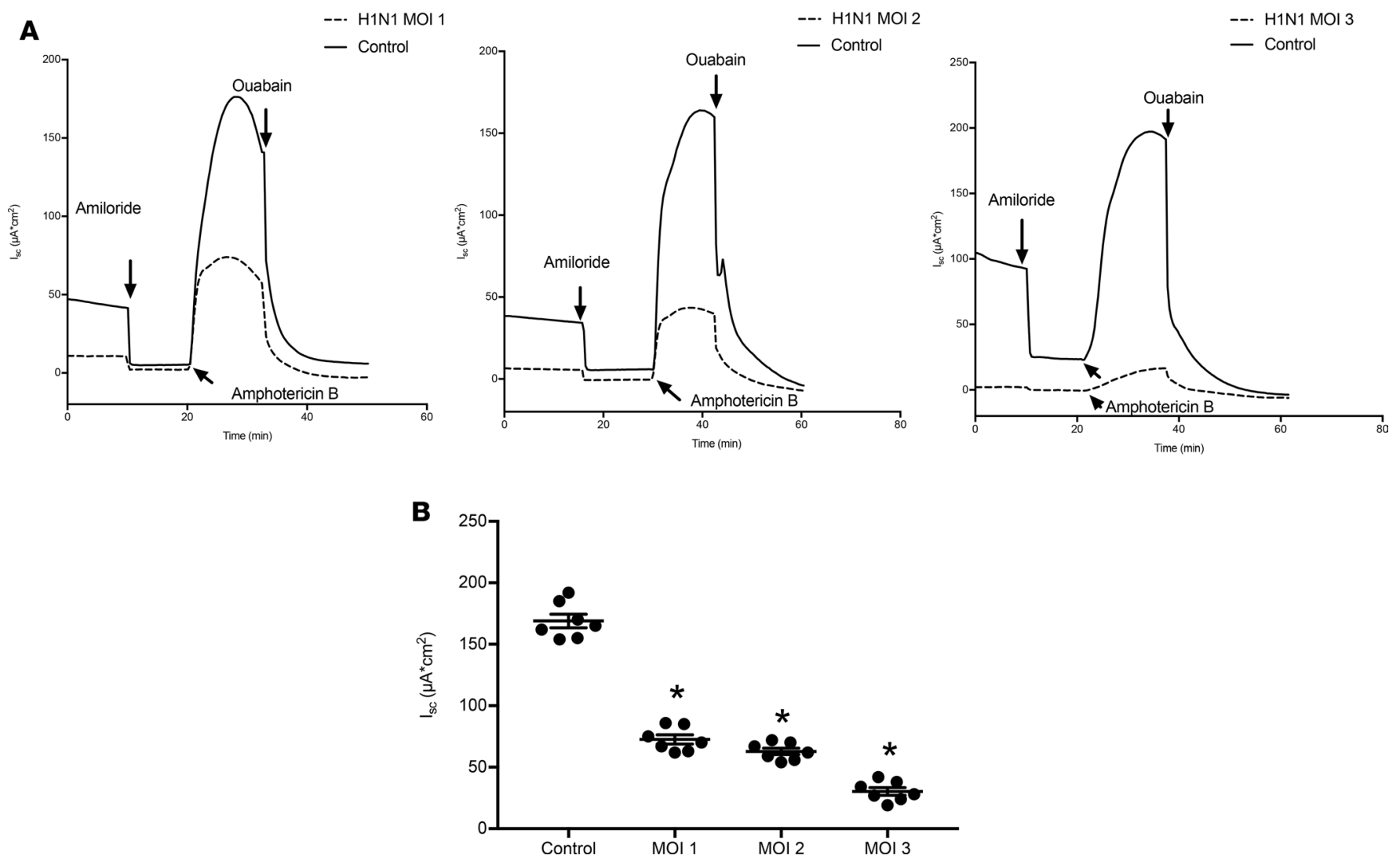

Figure 7. IAV decreases $\mathrm{Na}^{+} / \mathrm{K}^{+}-$ATPase activity. NHBEs were grown at an air-liquid interface and infected with A/California/07/2009 at MOls of 1, 2, and 3, and $\mathrm{Na}$ /K-ATPase activity was measured in an Ussing chamber system, following permeabilization of the apical membranes with nystatin. IAV caused reductions in $\mathrm{Na}$ /K-ATPase activity at all MOls tested. (A) Na/K-ATPase after IAV infection at MOls of 1, 2, and 3. (B) Ouabain-sensitive currents are plotted as mean $\pm \mathrm{SEM}, N=7$. Data were analyzed by 1 -way ANOVA and post hoc Tukey test for multiple comparisons. ${ }^{*} P<0.0001$ compared with noninfected controls.

In contrast, Aeffner et al. (39) noted that reduced CFTR activity in heterozygous $\mathrm{Cftr}^{+/-}$mice had reduced burden of disease after lethal IAV infection. The inconsistency between our findings and those reported by Aeffner may reflect differences between lethal and sublethal challenge doses, the different time points used, and the selectivity of the different measures used. These findings have a number of important implications. First, we have established that ion channel dysfunction by IAV can be measured using a clinically relevant, minimally invasive technique and thus could be applied to human IAV infection and patient management. Second, our findings show that ion channel dysfunction occurs in vivo and, that in the case of diminished CFTR function, persists beyond the acute infection period. This may have implications for persistent conditions in humans such as bronchitis that can persist after viral clearance. Collectively, our findings show that IAV decreases ion channel dysfunction to a clinically relevant extent in experimental infection.

Previous studies in cultured cell systems show active viral replication is critical for regulation of ENaC and CFTR $(25,40)$; however, it was unclear if only a subset of cells expressed diminished ion channel function. Single-cell analysis has recently emerged as an important approach for understanding the continuum of cellular responses in a tissue to a single stimulus, such as the range of responses to a pathogen. Herein, we have applied single-cell electrophysiology techniques to determine the ion conductance in infected and neighboring uninfected cells in vivo. Using fluorescence-encoded recombinant IAV, we found that $\mathrm{ENaC}$ dysfunction in the distal lung epithelium was limited to only fluorescent, infected epithelial cells, while uninfected cells were unchanged. Likewise, in differentiated airway epithelium, reduced CFTR conductance was limited to infected but not uninfected lung epithelium. An earlier investigation found similar reductions in ENaC sodium absorption but, contrary to our results, found evidence for increased CFTR $\mathrm{Cl}^{-}$secretion using measurements of alveolar fluid clearance, which reflects channel function indirectly (37). These findings will be important for correcting the channelopathy acquired in response to IAV and honing possible interventional strategies for restoring proper channel function. Targeting correction of diminished 

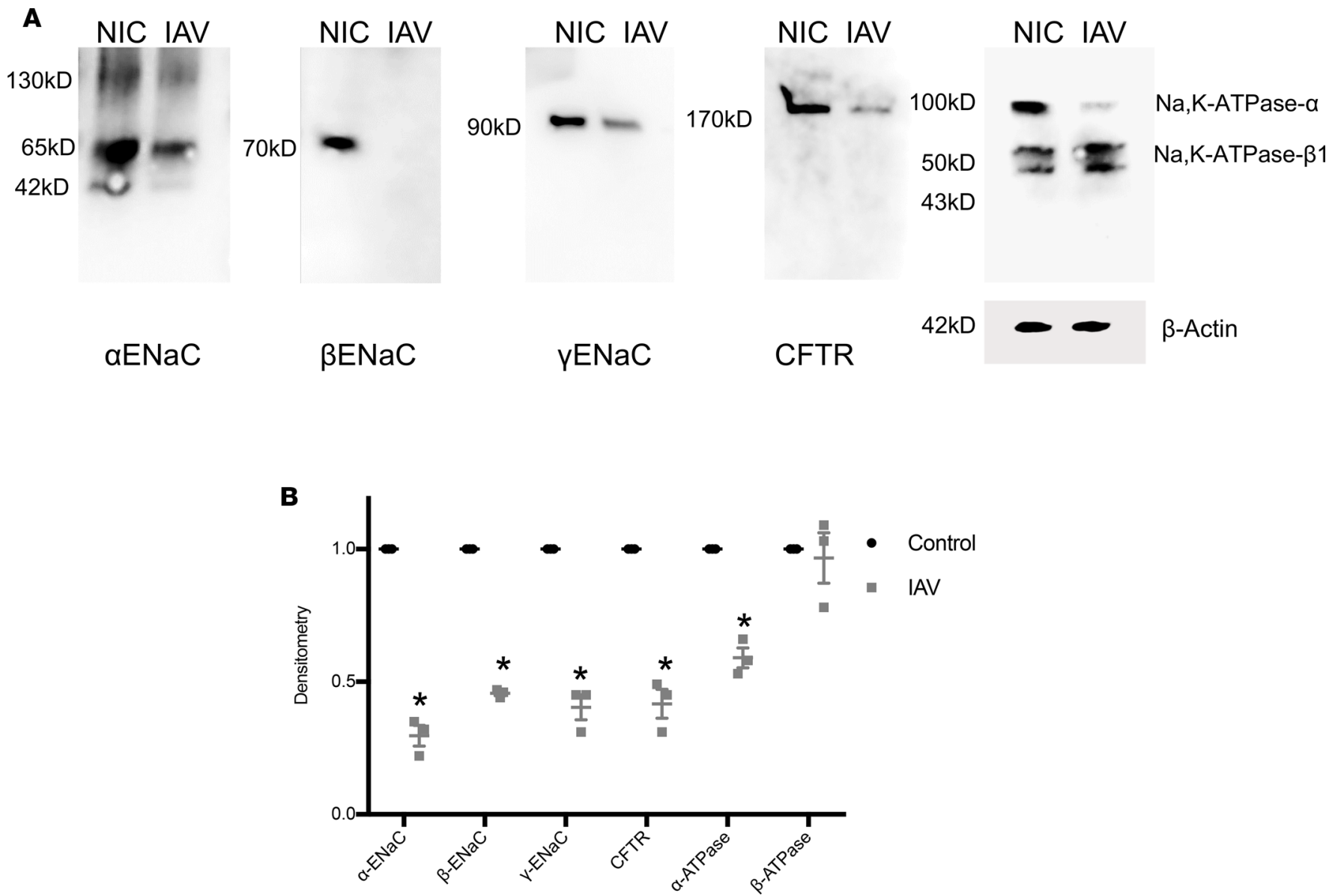

Figure 8. IAV decreases specific subunits of ENaC, CFTR, and Na/K-ATPase at the cell surface. NHBEs were grown at an air-liquid interface and infected with A/California/07/2009 at a MOI of 3. (A) ENaC, CFTR, and Na/K-ATPase protein abundance was measured by Western blotting. For ENaC and CFTR measurements, biotinylated apical membrane proteins were immunoblotted with specific antibodies against ENaC subunits and CFTR, respectively. Blots are representative of 3 separate experiments. (B) Densitometry demonstrates reductions in ENaC subunits, CFTR, and $\alpha$-Na/K-ATPase subunit, but no difference was observed in the expression of the regulatory $\beta$ subunit Na/K-ATPase levels. Data were analyzed by 1-way ANOVA and post hoc Tukey test for multiple comparisons. $N=3$ per group. ${ }^{*} P<0.001$ compared with mock-infected controls.

channel function in infected cells is an obvious interventional strategy and could hold promise for mitigating IAV pathogenesis. Indeed, we provide experimental evidence that correction of CFTR function can ameliorate airway pathophysiology after IAV. However, targeting the uninfected epithelium to increase channel function through increased gating time or increasing the number of channels could be a useful strategy as well. Thus, our findings here advance our knowledge regarding IAV regulation of the mucosal barrier and how targeting pharmacological approaches could be therapeutic.

Utilizing differentiated NHBEs grown at an air-liquid interface as a surrogate of human infection, we found that IAV infection downregulates the function of both ENaC and CFTR. These results are in agreement with previous reports using transformed cell lines $(24,25)$. We observed downregulation of CFTR and $\mathrm{ENaC}$ function only after 48 hours of infection in primary NHBEs that remained reduced throughout the study. In previous studies, we have shown that transfection of lung epithelial cells with M2, a hydrogen channel IAV protein that plays an essential role in IAV virus replication, downregulates both ENaC and CFTR by ubiquitinating these proteins and causing their removal from the cell surface $(24,25,41)$. In this study, both mouse-adapted $\mathrm{PR} 8^{\triangle \mathrm{GFP}}$ and human isolate $2009 \mathrm{pdmH} 1 \mathrm{~N} 1$ were able to reduce ENaC and CFTR function in both human and mouse primary epithelial ALI cultures. The difference in ENaC and CFTR activity was striking; however, it should be noted that, while transepithelial resistance was also significantly reduced, the short-circuit current recorded did not reflect a severely damaged monolayer or complete loss of the intracellular gradient driven by Na/K-ATPase activity. Immunoblotting analysis of $\mathrm{ENaC}$ subunits and CFTR revealed significant reductions in total protein from whole-cell homogenates and cell surface abundance of $\mathrm{ENaC} \beta$ and $\gamma$ subunit and CFTR, suggesting that IAV may regulate ion 
A

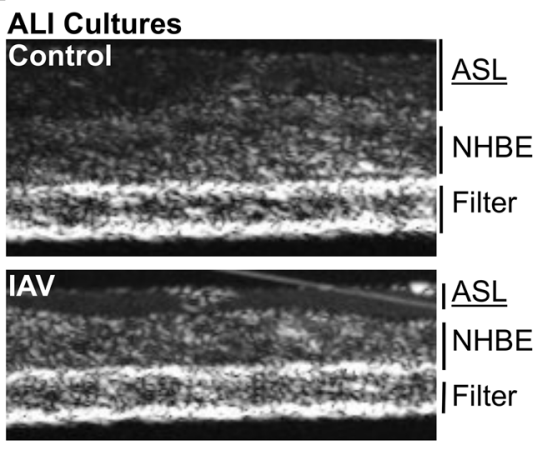

C

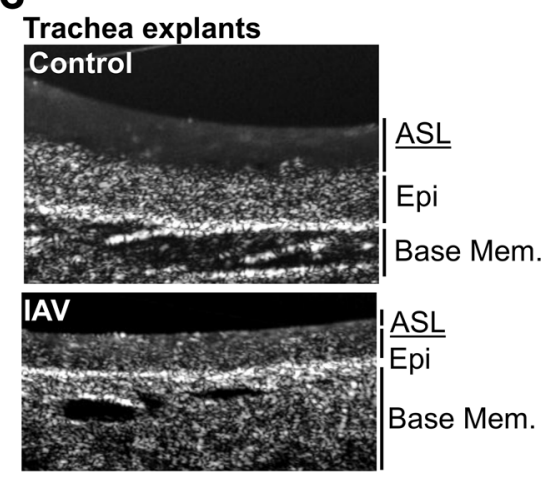

B
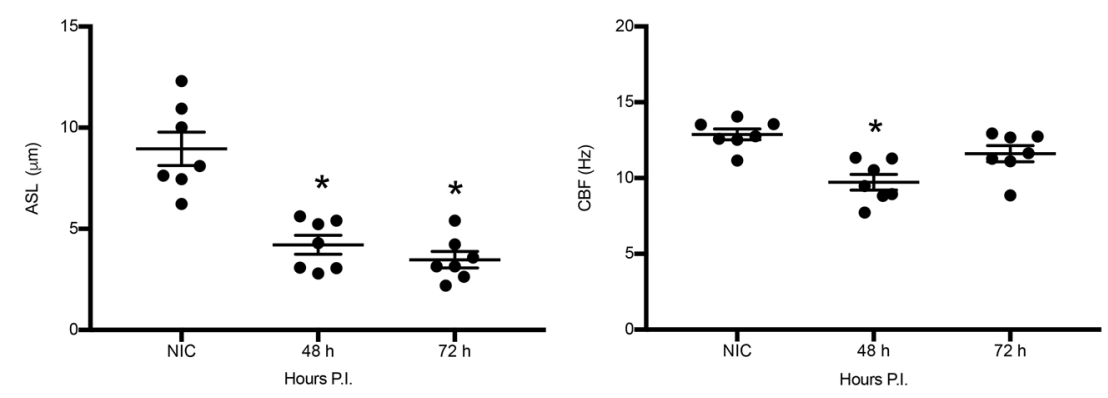

D

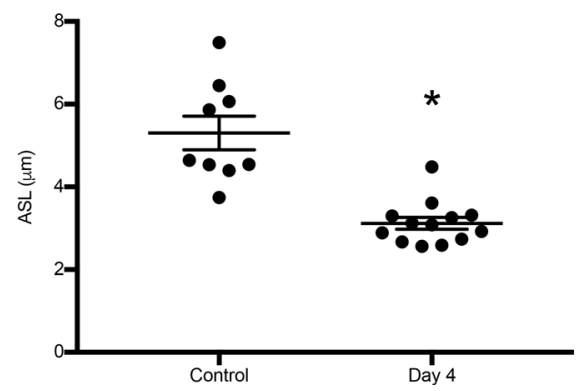

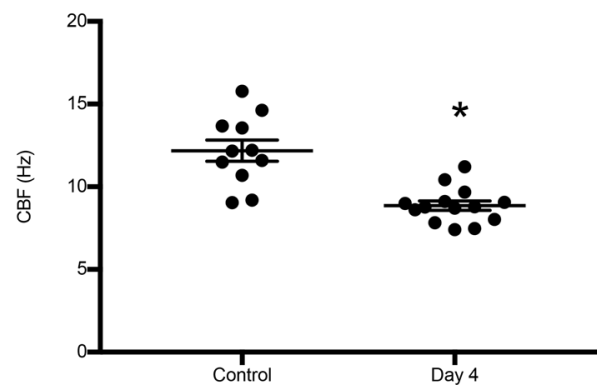

Figure 9. IAV infection reduces ASL depth and ciliary beat frequency in airway epithelial cultures and in vivo murine infection. NHBEs were grown at an air-liquid interface and infected with A/California/07/2009 at a MOI of 1 for 48 or 72 hours. ASL depth and CBF were imaged using $\mu$ OCT. (A) Representative OCT images from control or IAV-infected Transwell inserts identifying ASL, cell (NHBE), and filter structures. (B) IAV significantly $(P<0.003)$ reduced ASL depth and CBF within NHBE cultures at all time points and for the duration of experiments. (C) Representative images are displayed from $\mu$ OCT imaging of tracheas. (D) ASL and CBF in tracheas from infected mice were significantly decreased after 4 days of infection. (B and $\mathbf{D})$ Data, $n=7-13$ per group, are plotted as mean ASL depth or CBF \pm SEM. Data were analyzed by 1-way ANOVA and post hoc Tukey test for multiple comparisons. ${ }^{*} P<0.003$ compared with noninfected controls.

channel dysfunction through mechanisms related to protein trafficking or localization. Na/K-ATPase subunit $\alpha$ protein expression was also decreased by IAV infection which agrees with previous reports $(38,42)$. CFTR trafficking through the ER is highly regulated, as a number of genetic mutations in CFTR lead to ER retention or proteolysis due to misfolding (43). Deficits in ENaC abundance also occur due to an increase in ER stress $(44,45)$. IAV causes the unfolded protein response and ER stress $(46,47)$, both mechanisms that may result in reduction of ENaC and CFTR at the cell surface. Further studies are needed to more precisely elucidate the molecular mechanisms of IAV mediated on channel dysfunction.

A healthy airway mucosa is dependent on epithelial ion channel function to maintain ASL homeostasis (48). In CF, reduced CFTR function results in reduced airway hydration, reduced ASL $\mathrm{pH}$, reduced ciliary function, decreased mucus motility, and increased mucus $(14,49,50)$. As CFTR and ENaC function together to regulate the proper airway hydration (51), a loss in the function of both could result in an imbalance of airway $\mathrm{Na}^{+}$and $\mathrm{Cl}^{-}$, leading to altered mucus rheology. Using what we believe to be a novel imaging approach, we show here that IAV leads to a reduction in ASL depth and CBF in NHBE ALI cultures, similar to that seen in the CF airway. IAV infection in vivo leads to reduced ASL in excised tracheas analyzed ex vivo. Consistent with reduced ASL depth, CBF is reduced after IAV in vivo as well. Thus, both human and mouse models of IAV infection exhibit characteristics of airway pathophysiology consistent with reduced CFTR function. In addition, we show decrements in ASL depth can be restored by the use of the CFTR corrector lumacaftor, which acts to increase protein trafficking of CFTR through the ER. Correction of this defect with an FDA-approved drug may prove useful during severe IAV infections in reducing the incidence of ARDS and decreasing airway pathophysiology associated with IAV infection. Other CFTR potentiators or correctors are also available and approved (e.g., ivacaftor, tezacaftor). In light of our findings that cell surface level of CFTR are reduced by IAV, we would expect that CFTR correctors would 
A
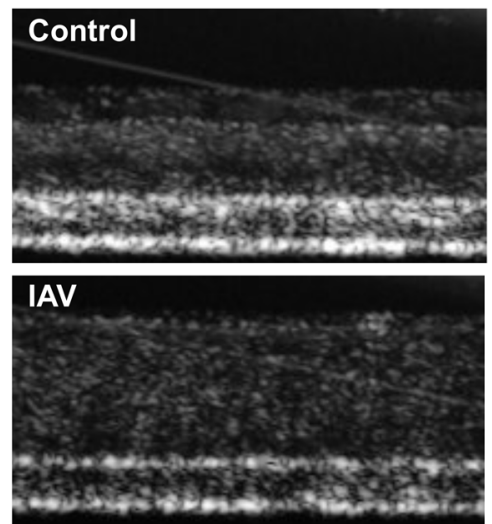

\section{ASL \\ NHBE \\ Filter}

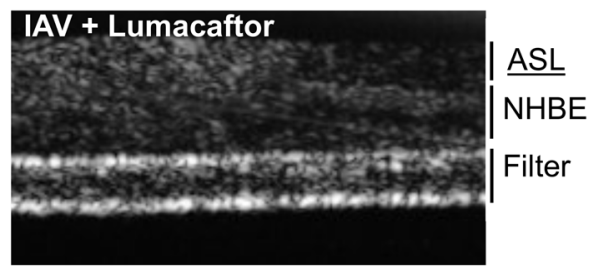

B

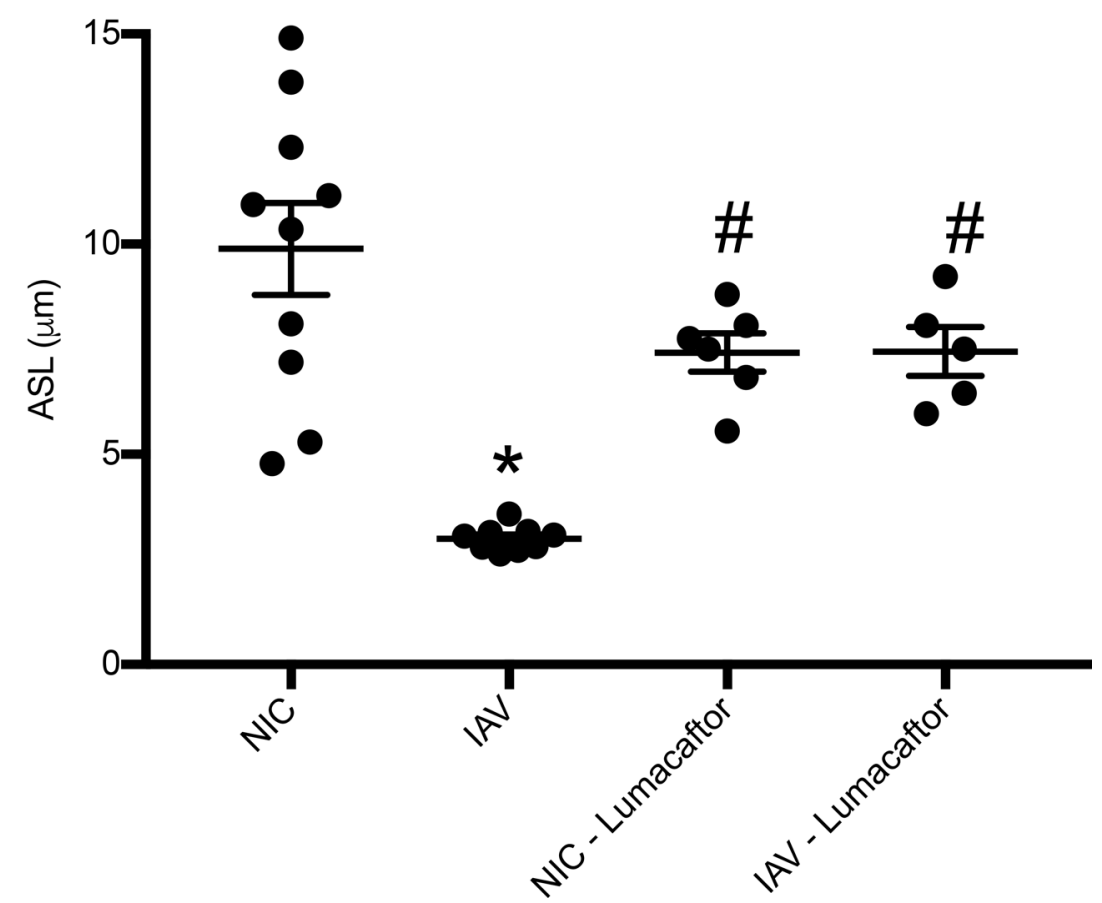

Figure 10. Pharmacological correction of CFTR restores ASL depth after IAV infection. NHBEs were grown at an air-liquid interface and infected with A/California/07/2009 at a MOI of 1 for 48 hours. Lumacaftor $(10 \mu \mathrm{M})$ was given via the basolateral compartment 1 hour p.i. ASL depth was measured by $\mu O C T$ imaging. (A) Representative images are displayed from $\mu$ OCT imaging of uninfected NHBEs, infected NHBEs, and infected NHBEs with lumacaftor treatment. (B) ASL was restored to uninfected levels after treatment with lumacaftor. Data are plotted as mean ASL depth \pm SEM, with $n=6-10$ per group across 2 donors. Data were analyzed by 1-way ANOVA with a post hoc Tukey test for multiple comparisons. ${ }^{*} P<0.0001$ compared with noninfected controls, ${ }^{\#} P<0.04$ compared with IAV.

likely be more efficacious than potentiators that work through increasing channel function through gating mechanisms. The diminution in ASL depth and CBF once again parallel both the time course of IAV infection and changes in ion transport. These data are in agreement with the other findings reported here and suggest that the ion channel dysfunction leads to changes in airway homeostasis and mucosal function.

The results herein demonstrate that IAV infection alters ion channel function of epithelial cells both in primary human airway epithelial cells and in vivo mouse models. Functionally, we found that IAV infection reduces ASL depth and $\mathrm{CBF}$ and that these changes were accompanied by downregulation of both ENaC and CFTR function. ASL depth is largely regulated by function of CFTR and ENaC, which are themselves regulated by one another (52). A downregulation of both channels might suggest a net zero change in ASL depth, as decreased ENaC function should increase ASL depth, whereas decreased CFTR function should decrease ASL depth. However, regulation of ASL depth is subject to regulation by not only ENaC and CFTR function, but also other ion channels and transporters. For example, previous studies showed that IAV infection reduced $\mathrm{Na}, \mathrm{K}$-ATPase in the plasma membrane of human and murine alveolar epithelial cells and in distal lung epithelium of infected mice, a host signaling pathway that involves epithelial type I IFN and an IFN-dependent elevation of macrophage TNF-related apoptosis-inducing ligand (TRAIL) (42). Our findings in polarized human airway cells mounting in Ussing chambers also show that influenza infection downregulates $\mathrm{Na}, \mathrm{K}$-ATPase activity. However, our patch-clamp measurements show that downregulation of ion channel activity only happens in infected cells, suggesting that ion channel activity is reduced irrespective of paracrine or other extrinsic factors. In addition, IAV is known to have profound effects on epithelial cells - dysregulating metabolism, protein synthesis, and immune function (42). Alteration of these normal cellular functions is likely to have a myriad of effects that require further investigation. Our findings presented here highlight the importance of ion channel dysfunction in IAV-mediated lung disease and suggest that acquired ENaC and CFTR dysfunction during IAV infection may be central to lung pathophysiology of barrier function. 


\section{Methods}

Virus. Madin-Darby canine kidney (MDCK, CCL-34) cells (ATCC) were maintained in minimum essential medium supplemented with L-glutamine, Earle's salts, 10\% fetal bovine serum, and $100 \mathrm{U} / \mathrm{ml}$ penicillin, and $100 \mu \mathrm{g} / \mathrm{ml}$ streptomycin (Antimycotic-Antibiotic; 15240-062 Gibco/Life Technologies Corp.). The IAV A/California/07/2009 H1N1 strain was obtained from the Centers for Disease Control. IAV A/ $\mathrm{PR} / 8 / 72$ (H1N1), referred to as PR8 $8^{\triangle \mathrm{GFP}}$ and designed to express a GFP-NS1 protein, was provided by A. García-Sastre (53). Viruses were propagated in MDCK cells. Titers of stock were measured using a standard plaque assay on 6-well plates of confluent monolayers of MDCK cells as described previously (54) using a $0.6 \%$ Avicel CL-611 solution (FMC Corporation) containing $3 \mu \mathrm{g} / \mathrm{ml}$ trypsin solution treated with 1-tosylamido-2-phenylethyl chloromethyl ketone (TPCK) (Worthington Biochemical Corporation). Viral stocks were stored in liquid nitrogen and thawed immediately before use.

Isolation and culture of differentiated human bronchial epithelial cells. Human bronchial epithelial cells were isolated from human lung tissue obtained from the organ and tissue donation program of the International Institute for the Advancement of Medicine using a previously published method (55). Cells isolated from human trachea and primary bronchi were plated onto collagen-coated 100-mm dishes (Biocoat; Becton Dickinson) and grown in BEGM medium (Lonza) to $80 \%$ confluency. Cells were then passaged onto 6.5-mm diameter Transwell inserts (0.4- $\mu \mathrm{m}$ pore size) treated with FNC Coating Mix (0407, AthenaES) and differentiated at an ALI using Lonza tissue culture reagents (Lonza) for bronchial epithelial cell growth and differentiation following the manufacturer's protocol. Cellular differentiation was enhanced, following exposure of the cells to air, by adding $10 \mathrm{nM}$ recombinant human neuregulin-1 $\alpha$ /epidermal growth factor-like domain of heregulin- $\alpha$ (296-HR-050, R\&D Systems) to the basolateral ALI media. After approximately 2 weeks of exposure to air, cells demonstrated hallmarks of differentiation (mucus production and appearance of ciliated cells).

Animals. Male and female 8- to 12-week-old C57B1/6 mice were purchased from Taconic Biosciences. For infections, mice were anesthetized with subcutaneous dexmedetomidine $(0.1 \mathrm{mg} / \mathrm{kg})$ and inhaled isoflurane (5\%) prior to intranasal instillation with 4,000 PFU PR8 ${ }^{\triangle \mathrm{GFP}}$ (total volume $50 \mu \mathrm{l}$ ) dissolved in PBS. Mice were monitored p.i. for weight, temperature, and clinical score once daily. Animals were then sacrificed at specified time points p.i. by pentobarbital overdose $(200 \mathrm{mg} / \mathrm{kg})$ (Euthasol, Virbac), and tissues were extracted for proceeding analysis.

NPD. NPD were measured with the technique published by Knowles and colleagues (12). Mice were anesthetized with $100 \mathrm{mg} / \mathrm{kg}$ ketamine and $0.5 \mathrm{mg} / \mathrm{kg}$ dexmedetomidine intraperitoneally. The nasal cannula was inserted to a distance of $3-5 \mathrm{~mm}$ from the nostril opening. A reference bridge was placed in the subcutaneous space of the hind leg. In mice, the perfusion rate was constant at $200 \mu 1 / \mathrm{h}$, just enough solution to connect the nasal epithelium to the recording amplifier (BMA-200 AC/DC Bioamplifier, CWE) without drowning the animal. The following protocol was followed during NPD measurements: after the mouse was placed on the experimental warming pad, after it was anesthetized and calm, the recording catheter was then inserted in one of the nostrils and sodium-driven NPD was recorded as a negative voltage deflection on the screen. Sodium-driven NPD was inhibited by switching microperfusion solution from Ringer's to Ringer's containing $200 \mu \mathrm{M}$ amiloride. To activate the chloride-driven NPD, the microperfusion was switched to Ringer's containing $20 \mu \mathrm{M}$ forskolin, the activation of CFTR in the nasal epithelium allows chloride secretion and thus a deflection of about -15 to $-20 \mathrm{mV}$ in a normal mouse. The chloride secretion through CFTR was then inhibited by CFTR inhibitor GLyH-101. GlyH-101 was used at a final concentration of $50 \mu \mathrm{M}$.

Lung slice patch clamps. Lungs of sacrificed mice were extracted from the thoracic cavity and perfused with PBS until white in appearance. Subsequently, the lungs were washed with Krebs-Ringer solution containing (in $\mathrm{mM}$ ) $140 \mathrm{NaCl}, 3 \mathrm{KCl}, 2.5 \mathrm{CaCl}_{2}, 1 \mathrm{MgCl}, 10$ glucose, and $10 \mathrm{HEPES}$ ( $\mathrm{pH} \approx 7.35-7.4$ ). Lung slices were prepared as previously reported (56). Lung slices were transferred to a chamber filled with a solution of the following composition (in $\mathrm{mM}$ ): $130 \mathrm{~K}$-gluconate, $2 \mathrm{MgCl}_{2}, 10 \mathrm{KCl}, 10$ glucose, and 10 HEPES (pH 7.4, KOH) and held in place with an anchor (Warner Instruments). The composition of the pipette solution was as follows (in $\mathrm{mM}$ ): $140 \mathrm{NaCl}, 3 \mathrm{KCl}, 2.5 \mathrm{CaCl}_{2}, 1 \mathrm{MgCl}, 5$ glucose, and $10 \mathrm{HEPES}$ $(\mathrm{pH}=7.4)$. For electrophysiology recordings, the chamber was placed on the recording stage of an Olympus microscope EX51WI (Olympus). Amiloride, forskolin, and GlyH-101 were applied to the cell patch through the pipette to minimize the damage to the high-resistance seal between the pipette and the cell membrane. Single-channel events were recorded using an Axopatch amplifier (Molecular Devices) interfaced with a computer by Digidata 1530 (Molecular Devices), an analog-to-digital and digital-to-analog converter. 
The data were recorded a sampling rate of $1 \mathrm{KHz}$ and filtered at $2 \mathrm{KHz}$. The data were stored to the hard drive or a PC running pClamp 10.6 (Molecular Devices). All data were analyzed using Clampfit (Molecular Devices), and the final figures and statistics were prepared using Igor Pro 7 (Wavemetrics).

Fluorescence microscopy. Mice were infected with $\mathrm{PR} 8^{\Delta \mathrm{GFP}}$ as outlined above. Lungs were removed, inflated with $1 \mathrm{ml}$ of a 1:1 mix of optical cutting temperature medium, and $2 \mathrm{M}$ sucrose. The lungs were then frozen on ice and sectioned on a cryostat at a thickness of $5 \mu \mathrm{m}$. Sections were placed on slides and mounted with DAPI Fluoromount-G (SouthernBiotech). Images were captured on a Leica DM5000B microscope using LASX v. 1.51 imaging software (Leica Microsystems).

Ussing chamber analysis. Cell monolayers were washed once with PBS, followed by incubation with either A/California/04/2009 or PR8 $8^{\triangle \mathrm{GFP}}$ virus at MOI of 1 or 3 or an equivalent volume of PBS for 1 hour. The monolayers were then aspirated and washed once with PBS. At the indicated time p.i., monolayers of NHBEs were mounted in EM-CSYS-8 Ussing chambers (Physiologic Instruments) and bathed on both sides with Ringers solutions containing (mM) $120 \mathrm{NaCl}, 25 \mathrm{NaHCO}_{3}, 3.3 \mathrm{KH}_{2} \mathrm{PO}_{4}, 0.83 \mathrm{~K}_{2} \mathrm{HPO}_{4}, 1.2$ $\mathrm{CaCl}_{2}, 1.2 \mathrm{MgCl}_{2}, 10 \mathrm{HEPES}\left(\mathrm{Na}^{+}\right.$-free), and 10 glucose (basolateral compartment). Osmolarity of all solutions, as measured by a freezing point depression osmometer (Advanced Instruments), was between 290 and $300 \mathrm{mOsm}$. Bath solutions were stirred vigorously by bubbling continuously with $95 \% \mathrm{O}_{2}, 5 \% \mathrm{CO}_{2}$ at $37^{\circ} \mathrm{C}$ ( $\mathrm{pH}$ 7.4). Monolayers were short circuited to $0 \mathrm{mV}$ and $\mathrm{I}_{\mathrm{sc}}$ were measured with an epithelial voltage clamp (VCC-MC8, Physiologic Instruments). A 10-mV pulse of 1-second duration was imposed every 10 seconds to monitor electrical resistance, which was calculated using Ohm's law. Data were collected using the Acquire and Analyze program, version 1.45 (Physiologic Instruments). Once $\mathrm{I}_{\text {sc }}$ reached steady-state values, amiloride $(10 \mu \mathrm{M})$ was added into the apical compartment to block ENaC activity. After $\mathrm{I}_{\mathrm{sc}}$ and electrical resistance reached new steady-state values, we added forskolin $(10 \mu \mathrm{M})$ to stimulate CFTR activation, followed by the specific CFTR inhibitor GlyH-101 $(50 \mu \mathrm{M})$.

Western blots. NHBEs grown at an air-liquid interface were infected with IAV A/California/07/2009 H1N1 at a MOI of 3 for 48 hours. Samples were then processed for detection of cell surface CFTR and ENaC and total cell lysate Na,K-ATPase. Cells were lysed with radio immunoprecipitation assay buffer. Cell surface proteins were prepared using the Pierce Cell Surface Protein Isolation kit following the manufacturer's instructions. Protein was loaded into an SDS-PAGE gel in equal quantities, and bands were detected with the follow antibodies: $\alpha$-ENaC (sc-20966), $\beta$-ENaC (sc-25354), and Na,K-ATPase $\beta 1$ (sc-21713) (all from Santa Cruz Biotechnology) and $\gamma$-ENaC (ab3468), CFTR (ab2478), and Na,K-ATPase $\alpha$ (ab76020) (all from Abcam).

$\mu O C T$ imaging. $\mu \mathrm{OCT}$, a high-speed and high-resolution interferometry-based reflectance imaging modality, was used to interrogate functional microanatomic parameters in primary cell cultures and excised tissue $(4,29,57)$. $\mu$ OCT possesses a roughly 1 -micron resolution, which is sufficient to fully resolve ASL depth and $\mathrm{CBF}$ without the use of exogenous dyes or particles. A full description of $\mu \mathrm{OCT}$ methods for airway epithelia functional microanatomy interrogation and quantitative image analysis is provided elsewhere (29). Images were acquired at a rate of 40 frames per second and at 512 lines per frame. ASL depths were quantitatively characterized by direct geometric measurements, and CBF was assessed over a time series of images. Each NHBE monolayer was imaged at 5 equally distributed regions of interest, $1 \mathrm{~mm}$ from the filter periphery, with a scanning beam parallel to the tangent of the circumference of the filter membrane disc.

Statistics. Grouped comparisons were made using 1-way or 2-way ANOVA with post hoc Tukey's or Bonferroni test. Single comparisons were performed using a 2-tailed Student's $t$ test. A $P$ value of less than 0.05 (2-tailed) was considered significant. All values are expressed as mean \pm SEM.

Study approval. The University of Alabama at Birmingham Institutional Animal Care and Use Committee approved all experimental procedures involving animals.

\section{Author contributions}

JDB, AL, SM, KSH, and SMR wrote and edited the manuscript and designed the experiments. JBD, AL, JET, RJS, ATA, JLT, ARA, and ZY performed experiments and analyzed data.

\section{Acknowledgments}

JDB, JET, JLT, AA, and KSH are supported by NIH grant R01 AI111475. RJS is supported by NIH grant T32HL105346 (PI Victor J. Thannickal). SMR is supported by NIH grants R35 HL135816 and P30 DK072482. SM and AL are supported by the CounterACT Program, NIH, Office of the Director and the National Institute of Environmental Health Sciences grants 5U01ES026458 02 and 1 U01 ES027697 01. 
Address correspondence to: Kevin S. Harrod, Department of Anesthesiology and Perioperative Medicine, School of Medicine, University of Alabama at Birmingham, BMR II 234, 901 19th St. South, Birmingham, Alabama, 35294, USA. Phone: 205.294.1929; Email: kevinharrod@uabmc.edu.

1. Louie JK, et al. Factors associated with death or hospitalization due to pandemic 2009 influenza A(H1N1) infection in California. JAMA. 2009;302(17):1896-1902.

2. Jain S, et al. Hospitalized patients with 2009 H1N1 influenza in the United States, April-June 2009. N Engl J Med. 2009;361(20):1935-1944.

3. Taylor G, et al. Epidemiological features of influenza in Canadian adult intensive care unit patients. Epidemiol Infect. 2016;144(4):741-750.

4. Birket SE, et al. Combination therapy with cystic fibrosis transmembrane conductance regulator modulators augment the airway functional microanatomy. Am J Physiol Lung Cell Mol Physiol. 2016;310(10):L928-L939.

5. Matalon S, Bartoszewski R, Collawn JF. Role of epithelial sodium channels in the regulation of lung fluid homeostasis. Am J Physiol Lung Cell Mol Physiol. 2015;309(11):L1229-L1238.

6. Gillie DJ, Pace AJ, Coakley RJ, Koller BH, Barker PM. Liquid and ion transport by fetal airway and lung epithelia of mice deficient in sodium-potassium-2-chloride transporter. Am J Respir Cell Mol Biol. 2001;25(1):14-20.

7. Åstrand $\mathrm{AB}$, et al. Linking increased airway hydration, ciliary beating, and mucociliary clearance through ENaC inhibition. $A m$ J Physiol Lung Cell Mol Physiol. 2015;308(1):L22-L32.

8. Sears PR, Yin WN, Ostrowski LE. Continuous mucociliary transport by primary human airway epithelial cells in vitro. Am J Physiol Lung Cell Mol Physiol. 2015;309(2):L99-108.

9. Kunzelmann K, Kathöfer S, Greger R. Na+ and Cl- conductances in airway epithelial cells: increased Na+ conductance in cystic fibrosis. Pflugers Arch. 1995;431(1):1-9.

10. O'Sullivan BP, Freedman SD. Cystic fibrosis. Lancet. 2009;373(9678):1891-1904.

11. Rowe SM, Miller S, Sorscher EJ. Cystic fibrosis. N Engl J Med. 2005;352(19):1992-2001.

12. Knowles MR, Paradiso AM, Boucher RC. In vivo nasal potential difference: techniques and protocols for assessing efficacy of gene transfer in cystic fibrosis. Hum Gene Ther. 1995;6(4):445-455.

13. Londino JD, Lazrak A, Collawn JF, Bebok Z, Harrod KS, Matalon S. Influenza virus infection alters ion channel function of airway and alveolar cells: mechanisms and physiological sequelae. Am J Physiol Lung Cell Mol Physiol. 2017;313(5):L845-L858.

14. Birket SE, et al. A functional anatomic defect of the cystic fibrosis airway. Am J Respir Crit Care Med. 2014;190(4):421-432.

15. Raju SV, et al. The cystic fibrosis transmembrane conductance regulator potentiator ivacaftor augments mucociliary clearance abrogating cystic fibrosis transmembrane conductance regulator inhibition by cigarette smoke. Am J Respir Cell Mol Biol. 2017;56(1):99-108.

16. Lazrak A, et al. Enhancement of alveolar epithelial sodium channel activity with decreased cystic fibrosis transmembrane conductance regulator expression in mouse lung. Am J Physiol Lung Cell Mol Physiol. 2011;301(4):L557-L567.

17. Lazrak A, et al. Regulation of alveolar epithelial Na+ channels by ERK1/2 in chlorine-breathing mice. Am J Respir Cell Mol Biol. 2012;46(3):342-354.

18. Thibodeau PH, Butterworth MB. Proteases, cystic fibrosis and the epithelial sodium channel (ENaC). Cell Tissue Res. 2013;351(2):309-323.

19. Gaillard EA, Kota P, Gentzsch M, Dokholyan NV, Stutts MJ, Tarran R. Regulation of the epithelial Na+ channel and airway surface liquid volume by serine proteases. Pflugers Arch. 2010;460(1):1-17.

20. Novak I. Purinergic signalling in epithelial ion transport: regulation of secretion and absorption. Acta Physiol (Oxf). 2011;202(3):501-522.

21. Helms MN, Jain L, Self JL, Eaton DC. Redox regulation of epithelial sodium channels examined in alveolar type 1 and 2 cells patch-clamped in lung slice tissue. J Biol Chem. 2008;283(33):22875-22883.

22. Peters-Hall JR, et al. Long-term culture and cloning of primary human bronchial basal cells that maintain multipotent differentiation capacity and CFTR channel function. Am J Physiol Lung Cell Mol Physiol. 2018;315(2):L313-L327.

23. Thome U, et al. Novel SIN-1 reactive intermediates modulate chloride secretion across murine airway cells. Free Radic Biol Med. 2003;35(6):662-675

24. Londino JD, Lazrak A, Jurkuvenaite A, Collawn JF, Noah JW, Matalon S. Influenza matrix protein 2 alters CFTR expression and function through its ion channel activity. Am J Physiol Lung Cell Mol Physiol. 2013;304(9):L582-L592.

25. Londino JD, et al. Influenza virus M2 targets cystic fibrosis transmembrane conductance regulator for lysosomal degradation during viral infection. FASEB J. 2015;29(7):2712-2725.

26. Guo Y, DuVall MD, Crow JP, Matalon S. Nitric oxide inhibits Na+ absorption across cultured alveolar type II monolayers. $A m$ J Physiol. 1998;274(3 Pt 1):L369-L377.

27. Chen L, Patel RP, Teng X, Bosworth CA, Lancaster JR, Matalon S. Mechanisms of cystic fibrosis transmembrane conductance regulator activation by S-nitrosoglutathione. J Biol Chem. 2006;281(14):9190-9199.

28. Thome $\mathrm{U}$, et al. Na,K-ATPase gene transfer mitigates an oxidant-induced decrease of active sodium transport in rat fetal ATII cells. Am J Respir Cell Mol Biol. 2001;24(3):245-252.

29. Liu L, et al. Method for quantitative study of airway functional microanatomy using micro-optical coherence tomography. PLoS ONE. 2013;8(1):e54473.

30. Birket SE, et al. Combination therapy with cystic fibrosis transmembrane conductance regulator modulators augment the airway functional microanatomy. Am J Physiol Lung Cell Mol Physiol. 2016;310(10):L928-L939.

31. Birket SE, et al. Development of an airway mucus defect in the cystic fibrosis rat. JCI Insight. 2018;3(1):97199.

32. Raju SV, et al. Cigarette smoke induces systemic defects in cystic fibrosis transmembrane conductance regulator function. $A m J$ Respir Crit Care Med. 2013;188(11):1321-1330. 
33. Hudock KM, Clancy JP. An update on new and emerging therapies for cystic fibrosis. Expert Opin Emerg Drugs. 2017;22(4):331-346. 34. Deeks ED. Lumacaftor/ivacaftor: a review in cystic fibrosis. Drugs. 2016;76(12):1191-1201.

35. Chen XJ, et al. Influenza virus inhibits ENaC and lung fluid clearance. Am J Physiol Lung Cell Mol Physiol. 2004;287(2):L366-L373.

36. Rowe SM, Clancy JP, Wilschanski M. Nasal potential difference measurements to assess CFTR ion channel activity. Methods Mol Biol. 2011;741:69-86.

37. Wolk KE, et al. Influenza A virus inhibits alveolar fluid clearance in BALB/c mice. Am J Respir Crit Care Med. 2008;178(9):969-976.

38. Chan MC, et al. Human mesenchymal stromal cells reduce influenza A H5N1-associated acute lung injury in vitro and in vivo. Proc Natl Acad Sci USA. 2016;113(13):3621-3626.

39. Aeffner F, et al. Heterozygosity for the F508del mutation in the cystic fibrosis transmembrane conductance regulator anion channel attenuates influenza severity. J Infect Dis. 2013;208(5):780-789.

40. Chen $\mathrm{L}$, et al. Inhibition of $\mathrm{Na}+$ transport in lung epithelial cells by respiratory syncytial virus infection. Am J Respir Cell Mol Biol. 2009;40(5):588-600.

41. Lazrak A, Iles KE, Liu G, Noah DL, Noah JW, Matalon S. Influenza virus M2 protein inhibits epithelial sodium channels by increasing reactive oxygen species. FASEB J. 2009;23(11):3829-3842.

42. Peteranderl C, et al. Macrophage-epithelial paracrine crosstalk inhibits lung edema clearance during influenza infection. $J C l i n$ Invest. 2016;126(4):1566-1580.

43. Kim SJ, Skach WR. Mechanisms of CFTR folding at the endoplasmic reticulum. Front Pharmacol. 2012;3:201.

44. Crambert G, et al. Epithelial sodium channel abundance is decreased by an unfolded protein response induced by hyperosmolality. Physiol Rep. 2014;2(11):e12169.

45. Faria D, et al. Regulation of ENaC biogenesis by the stress response protein SERP1. Pflugers Arch. 2012;463(6):819-827.

46. Hassan IH, et al. Influenza A viral replication is blocked by inhibition of the inositol-requiring enzyme 1 (IRE1) stress pathway J Biol Chem. 2012;287(7):4679-4689.

47. Frabutt DA, Wang B, Riaz S, Schwartz RC, Zheng YH. Innate sensing of influenza a virus hemagglutinin glycoproteins by the host endoplasmic reticulum (ER) stress pathway triggers a potent antiviral response via ER-associated protein degradation. J Virol. 2018;92(1):e01690-17

48. Guidot DM, Folkesson HG, Jain L, Sznajder JI, Pittet JF, Matthay MA. Integrating acute lung injury and regulation of alveolar fluid clearance. Am J Physiol Lung Cell Mol Physiol. 2006;291(3):L301-L306.

49. Shah VS, et al. Airway acidification initiates host defense abnormalities in cystic fibrosis mice. Science. 2016;351(6272):503-507

50. Livraghi-Butrico A, et al. Contribution of mucus concentration and secreted mucins Muc5ac and Muc5b to the pathogenesis of muco-obstructive lung disease. Mucosal Immunol. 2017;10(3):829.

51. Berdiev BK, Qadri YJ, Benos DJ. Assessment of the CFTR and ENaC association. Mol Biosyst. 2009;5(2):123-127.

52. Stutts MJ, Rossier BC, Boucher RC. Cystic fibrosis transmembrane conductance regulator inverts protein kinase A-mediated regulation of epithelial sodium channel single channel kinetics. J Biol Chem. 1997;272(22):14037-14040.

53. Manicassamy B, Manicassamy S, Belicha-Villanueva A, Pisanelli G, Pulendran B, García-Sastre A. Analysis of in vivo dynamics of influenza virus infection in mice using a GFP reporter virus. Proc Natl Acad Sci USA. 2010;107(25):11531-11536

54. Matrosovich M, Matrosovich T, Garten W, Klenk HD. New low-viscosity overlay medium for viral plaque assays. Virol J. 2006;3:63

55. Fulcher ML, Gabriel S, Burns KA, Yankaskas JR, Randell SH. Well-differentiated human airway epithelial cell cultures. Methods Mol Med. 2005;107:183-206.

56. Bourke S, Mason HS, Borok Z, Kim KJ, Crandall ED, Kemp PJ. Development of a lung slice preparation for recording ion channel activity in alveolar epithelial type I cells. Respir Res. 2005;6:40.

57. Birket SE, et al. A functional anatomic defect of the cystic fibrosis airway. Am J Respir Crit Care Med. 2014;190(4):421-432. 